\title{
Astrocytic Ephrin-B1 Controls Excitatory-Inhibitory Balance in Developing Hippocampus
}

\author{
${ }^{1}$ Amanda Q. Nguyen, ${ }^{1,2}$ Samantha Sutley, ${ }^{1}$ Jordan Koeppen, ${ }^{1,3}$ Karen Mina, ${ }^{1}$ Simone Woodruff, ${ }^{1}$ \\ ${ }^{\circledR}$ Sandy Hanna, ${ }^{1}$ Alekya Vengala, ${ }^{1}$ Peter W. Hickmott, ${ }^{2,4}{ }^{\circledR}$ Andre Obenaus, ${ }^{5}$ and ${ }^{\circledR}$ Iryna M. Ethell ${ }^{1,2,3}$ \\ ${ }^{1}$ Division of Biomedical Sciences, University of California Riverside School of Medicine, Riverside, California $92521,{ }^{2}$ Neuroscience Graduate \\ Program, University of California Riverside, Riverside, California $92521,{ }^{3}$ Cell, Molecular, and Developmental Biology Graduate Program, University \\ of California Riverside, California 92521, ${ }^{4}$ Department of Psychology, University of California Riverside, Riverside, California 92521, and \\ ${ }^{5}$ Department of Pediatrics, University of California Irvine, Irvine, California 92350
}

Astrocytes are implicated in synapse formation and elimination, which are associated with developmental refinements of neuronal circuits. Astrocyte dysfunctions are also linked to synapse pathologies associated with neurodevelopmental disorders and neurodegenerative diseases. Although several astrocyte-derived secreted factors are implicated in synaptogenesis, the role of contact-mediated glial-neuronal interactions in synapse formation and elimination during development is still unknown. In this study, we examined whether the loss or overexpression of the membrane-bound ephrin-B1 in astrocytes during postnatal day (P) 14-28 period would affect synapse formation and maturation in the developing hippocampus. We found enhanced excitation of CA1 pyramidal neurons in astrocyte-specific ephrin-B1 KO male mice, which coincided with a greater vGlut1/PSD95 colocalization, higher dendritic spine density, and enhanced evoked AMPAR and NMDAR EPSCs. In contrast, EPSCs were reduced in CA1 neurons neighboring ephrin-B1-overexpressing astrocytes. Overexpression of ephrin-B1 in astrocytes during P14-28 developmental period also facilitated evoked IPSCs in CA1 neurons, while evoked IPSCs and miniature IPSC amplitude were reduced following astrocytic ephrin-B1 loss. Lower numbers of parvalbumin-expressing cells and a reduction in the inhibitory VGAT/gephyrin-positive synaptic sites on CA1 neurons in the stratum pyramidale and stratum oriens layers of KO hippocampus may contribute to reduced inhibition and higher excitation. Finally, dysregulation of excitatory/inhibitory balance in KO male mice is most likely responsible for impaired sociability observed in these mice. The ability of astrocytic ephrin-B1 to influence both excitatory and inhibitory synapses during development can potentially contribute to developmental refinement of neuronal circuits.

Key words: astrocyte; development; ephrin; excitatory-inhibitory balance; hippocampus; synapse

Significance Statement

This report establishes a link between astrocytes and the development of excitatory and inhibitory balance in the mouse hippocampus during early postnatal development. We provide new evidence that astrocytic ephrin-B1 differentially regulates development of excitatory and inhibitory circuits in the hippocampus during early postnatal development using a multidisciplinary approach. The ability of astrocytic ephrin-B1 to influence both excitatory and inhibitory synapses during development can potentially contribute to developmental refinement of neuronal circuits and associated behaviors. Given widespread and growing interest in the astrocyte-mediated mechanisms that regulate synapse development, and the role of EphB receptors in neurodevelopmental disorders, these findings establish a foundation for future studies of astrocytes in clinically relevant conditions.

Received Feb. 19, 2020; revised June 8, 2020; accepted July 20, 2020.

Author contributions: A.Q.N., S.S., P.W.H., and I.M.E. designed research; A.Q.N., S.S., J.K., K.M., S.W., S.H., A.V., and I.M.E. performed research; A.Q.N., S.S., J.K., K.M., S.W., S.H., A.V., P.W.H., A.O., and I.M.E. analyzed data; A.Q.N. and I.M.E. wrote the first draft of the paper; A.Q.N., S.S., J.K., K.M., S.W., P.W.H., A.O., and I.M.E. edited the paper; A.Q.N., S.S., and I.M.E. wrote the paper; J.K. contributed unpublished reagents/analytic tools.

This work was supported by National Institute of Mental Health Grant MH67121 to I.M.E. and National Institutes of Health Research Infrastructure Programs Grant 1S100D020042-01. We thank members of the
I.M.E., A.O., and P.W.H. laboratories for helpful discussions and comments; Arnold Palacios, Dr. Roman Vlkolinsky, Emil Rudobeck, Jeffrey Rumschlag, Micah Feri, Alexander King, and Mary Hamer for technical support; and David Carter for advice on confocal microscopy.

The authors declare no competing financial interests.

Correspondence should be addressed to Iryna M. Ethell at iryna.ethell@medsch.ucr.edu.

https://doi.org/10.1523/JNEUROSCI.0413-20.2020

Copyright $\odot 2020$ the authors 


\section{Introduction}

Synapses are the building blocks of neuronal networks functioning as fundamental information-processing units in the brain (Südhof and Malenka, 2008; Mayford et al., 2012). Excitatory glutamatergic synapses are specialized cell-cell connections that facilitate neuronal activity, which is also fine-tuned by a complex network of inhibitory inputs from GABA-containing interneurons. Activity-mediated formation, pruning, and maturation of specific synapses are important in establishing neural circuits. Improper synapse development that leads to imbalance between excitatory and inhibitory (E/I) synaptic activity is linked to several neurologic disorders, including autism spectrum disorders (ASDs) (Gao and Penzes, 2015; Lee et al., 2017) and epilepsy (Fritschy, 2008; Bonansco and Fuenzalida, 2016). Thus, investigations of the mechanisms underlying E/I synapse development may contribute to an understanding of the pathophysiological mechanisms of these brain disorders.

Astrocytes are able to control the neuronal circuits by regulating the formation, pruning, and maturation of synapses. Astrocyte-secreted factors, such as thrombospondin (Christopherson et al., 2005), hevin (Kucukdereli et al., 2011), and glypican (Allen et al., 2012), are known to promote synaptogenesis; whereas the release of gliotransmitters, such as glutamate (Fellin et al., 2004), D-serine (Henneberger et al., 2010), and TNF- $\alpha$ (Beattie et al., 2002; Stellwagen and Malenka, 2006), can modulate synaptic functions. Astrocytic processes are also suggested to modulate synapse number and function through direct contact with dendritic spines and presynaptic boutons (Araque et al., 1999; Ullian et al., 2001; Hama et al., 2004; Clarke and Barres, 2013; Chung et al., 2013; Allen and Eroglu, 2017).

EphB receptor tyrosine kinases and their ephrin-B ligands are membrane-associated proteins that play an important role in regulating cell-cell interactions during development, including axon guidance (Zimmer et al., 2003), spinogenesis, and synaptogenesis (Dalva et al., 2000; Ethell et al., 2001; Henkemeyer et al., 2003; Moeller et al., 2006; Segura et al., 2007). The trans-synaptic Eph/ephrin-B interactions can result in bidirectional signaling, activating forward signaling in the Eph-expressing cell, and reverse signaling in the ephrin-expressing cell (Bush and Soriano, 2009; Sloniowski and Ethell, 2012; Xu and Henkemeyer, 2012) that can promote postsynaptic spine formation and maturation during development (Henderson et al., 2001; Henkemeyer et al., 2003; Kayser et al., 2006). EphB receptors are known to directly interact with NMDARs and are important for the recruitment and retention of NMDARs at the synaptic site and modulating their function (Dalva et al., 2000; Henderson et al., 2001; Kayser et al., 2006; Nolt et al., 2011). EphB/ephrinB signaling can also influence synapse formation and maturation by regulating AMPARs at postsynaptic sites (Kayser et al., 2006; Hussain et al., 2015).

Our previous study suggests that the changes in ephrin-B1 levels in astrocytes may influence trans-synaptic interactions between neuronal ephrin-B and its EphB receptors, affecting synapse maintenance in the adult hippocampus (Koeppen et al., 2018). The goal of this study was to determine whether $\mathrm{KO}$ or overexpression (OE) of astrocytic ephrin-B1 would affect synapse formation and maturation in the developing hippocampus. Ephrin-B1 $\mathrm{KO}$ and $\mathrm{OE}$ were accomplished in astrocytes during the postnatal day (P) 14-28 developmental period of hippocampal synaptogenesis. Activity of CA1 hippocampal neurons was measured using both extracellular field recordings and wholecell patch-clamp electrophysiology to determine E/I synaptic changes. To evaluate E/I circuit changes, we further analyzed dendritic spine density and morphology, and the density of E/I synapses by immunohistochemistry through the analysis of vesicular glutamate transporter 1 (vGlut1)/postsynaptic density-95 (PSD95), vGlut1/PV, and VGAT/gephyrin-positive puncta. To examine the functional significance of the synaptic changes in ephrin-B1 KO mice, mouse behaviors, such as sociability, social novelty, anxiety, and hyperactivity, were also evaluated. We observed enhanced excitation of CA1 pyramidal neurons in the developing hippocampus of astrocyte-specific ephrin-B1 KO mice. Based on our new finding, we propose a new role of astrocytic ephrin-B1 in the development of both E/I circuits in CA1 hippocampus during P14-P28. The dysregulation of E/I balance induced by astrocyte-specific deletion of ephrin-B1 in the developing hippocampus is most likely responsible for impaired sociability observed in these mice.

\section{Materials and Methods}

Ethics statement. All mouse studies were done according to National Institutes of Health and Institutional Animal Care and Use Committee at the University of California Riverside (approval \#20190015 and \#20190029) guidelines; animal welfare assurance \#A3439-01 is on file with the Office of Laboratory Animal Welfare. Mice were maintained in an Association for Assessment and Accreditation of Laboratory Animal Care-accredited facility under $12 \mathrm{~h}$ light/dark cycle and fed standard mouse chow.

Mice. To achieve specific ephrin-B1 deletion in astrocytes, three different mouse lines were generated. In Group 1, ERT2-Cre ${ }^{\text {GFAP }}$ (B6.Cg$\mathrm{Tg}$ (GFAP-cre/ERT2)505Fmv/J RRID:IMSR_JAX:012849) male mice were crossed with ephrin-B1 $1^{\text {flox/+ }}$ (129S-Efnb1 ${ }^{\text {flox/+ }} /$ J, RRID:IMSR_JAX: 007664) female mice to obtain either ERT2-Cre ${ }^{G F A P}$ ephrin-B1 $1^{\text {flox } / y} \mathrm{KO}$ or ERT2-Cre ${ }^{G F A P}$ control male mice. In Group 2, ERT2-Cre ${ }^{G F A P}$ mice were first crossed with Rosa-CAG-LSL-tdTomato reporter mice (CAG-tdTomato; RRID:IMSR_JAX:007909) to generate tdTomatoERT2-Cre ${ }^{G F A P}$ mice. Then, tdTomatoERT2-Cre ${ }^{G F A P}$ male mice were crossed with ephrin-B1 $1^{\text {flox/+ }}$ female mice to obtain either tdTomatoERT2-Cre ${ }^{G F A P}$ ephrin-B1 $1^{\text {flox/y }}$ KO or tdTomatoERT2-Cre ${ }^{G F A P}$ control male mice, allowing for tdTomato expression in astrocytes and analysis of ephrin-B1 levels. In Group 3, ERT2-Cre ${ }^{\text {GFAP }}$ mice were first crossed with Thy1-GFP-M mice (Tg(Thy1-EGFP)MJrs/J, RRID:IMSR_JAX: 007788) to obtain Thy1-GFP-ERT2-Cre ${ }^{\text {GFAP }}$ mice. Then, Thy1-GFPERT2-Cre ${ }^{\text {GFAP }}$ male mice were crossed with ephrin- $B 1^{\text {flox } /+}$ female mice to obtain either Thy1-GFP-ERT2-Cre ${ }^{G F A P}$ ephrin-B1 ${ }^{\text {flox/y }}$ KO or Thy1GFP-ERT2-Cre ${ }^{\text {GFAP }}$ control male mice, allowing for GFP expression in excitatory pyramidal neurons for analysis of dendritic spines and synapses. Real-time PCR-based analysis of genomic DNA isolated from mouse tails was used to confirm genotypes by Transnetyx.

In all groups of mice, $\mathrm{KO}$ and their littermate control mice received tamoxifen at P14 intraperitoneally $(0.5 \mathrm{mg}$ in $5 \mathrm{mg} / \mathrm{ml}$ of $1: 9$ ethanol/ sunflower seed oil solution) once a day for 5 consecutive days, and analysis was performed at P28 (see Fig. 1A). Group 1 was used for electrophysiology, immunohistochemistry, Western blot, and behavioral analysis; Group 2 was used for immunohistochemical analysis of ephrinB1 expression levels; and Group 3 was used for dendritic spine and synapse analysis. To confirm specific ablation of ephrin-B1 in astrocytes, ephrin-B1 immunoreactivity was analyzed in the CA1 hippocampus (see Fig. $1 B$ ) of mouse Group 1 (ERT2-Cre ${ }^{G F A P}$ ephrin- $B 1^{\text {flox/y }} \mathrm{KO}$ and their control littermates) and Group 2 (tdTomatoERT2-Cre ${ }^{G F A P}$ ephrin-B1 ${ }^{\text {flox/y }}$ $\mathrm{KO}$; see Fig. 1C,D). Ephrin-B1 immunoreactivity was significantly reduced in hippocampal astrocytes of tamoxifen-treated KO mice (see Fig. 1E).

To achieve OE of ephrin-B1 in hippocampal astrocytes, we used adeno-associated viral particles (VPs) containing ephrin-B1 cDNA under GFAP promoter to ensure specific expression of ephrin-B1 in astrocytes (AAV7.GfaABC1D.ephrin-B1.SV40), which is referenced in the text as AAV-ephrin-B1. Control AAV-tdTomato VP contained tdTomato cDNA under the same GFAP promoter (AAV7.GfaABC1D. tdTomato.SV40). Experimental AAV-ephrin-B1 VP (final concentration 
A Developmental period P14-28

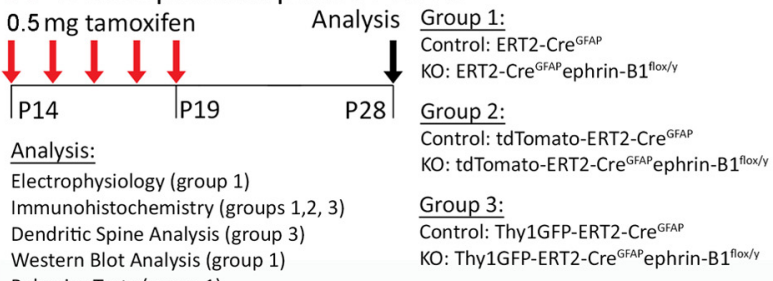

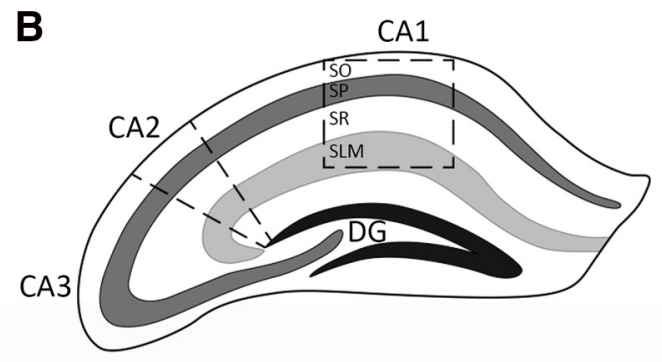

C ephrin-B1 tdTomato DAPI
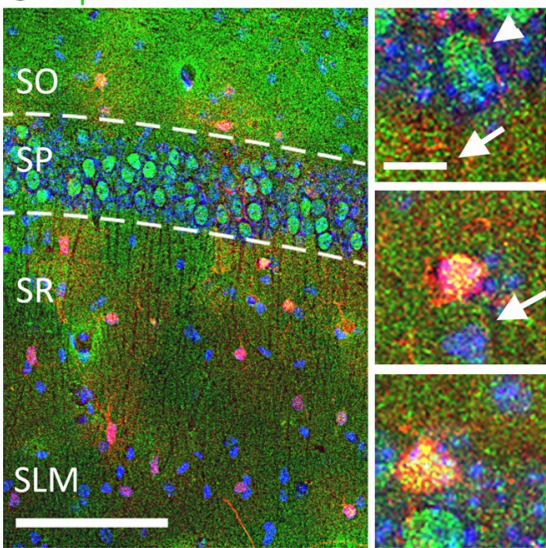

Control D

ephrin-B1 tdTomato DAPI
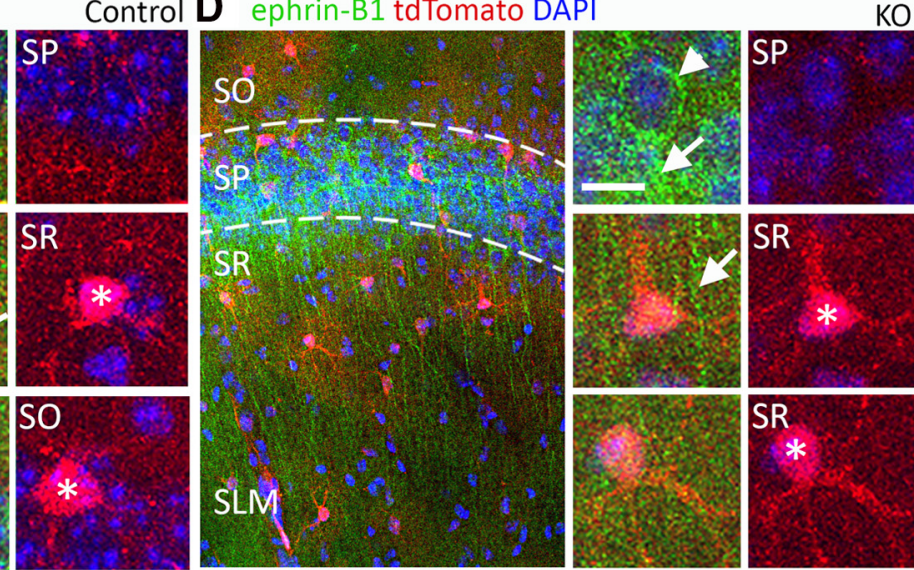

E

Ephrin-B1 levels
in astrocytes

\section{Ephrin-B1 in
cell body}

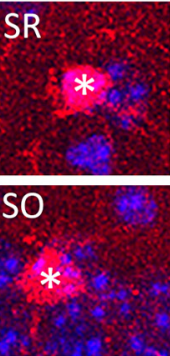

\section{F}

F Extracellular Field Recordings
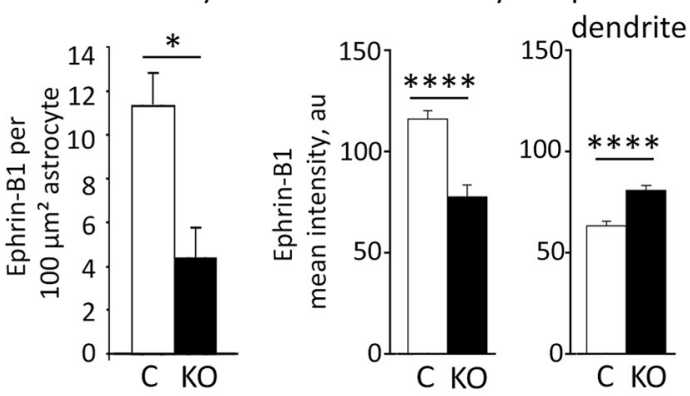

\section{G fEPSP}

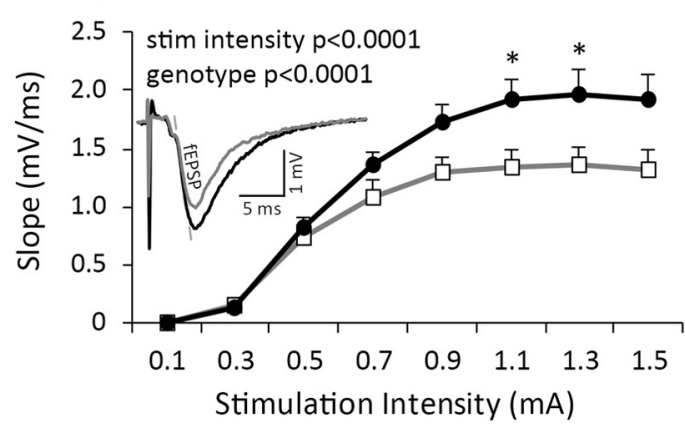

\section{H Population Spike}
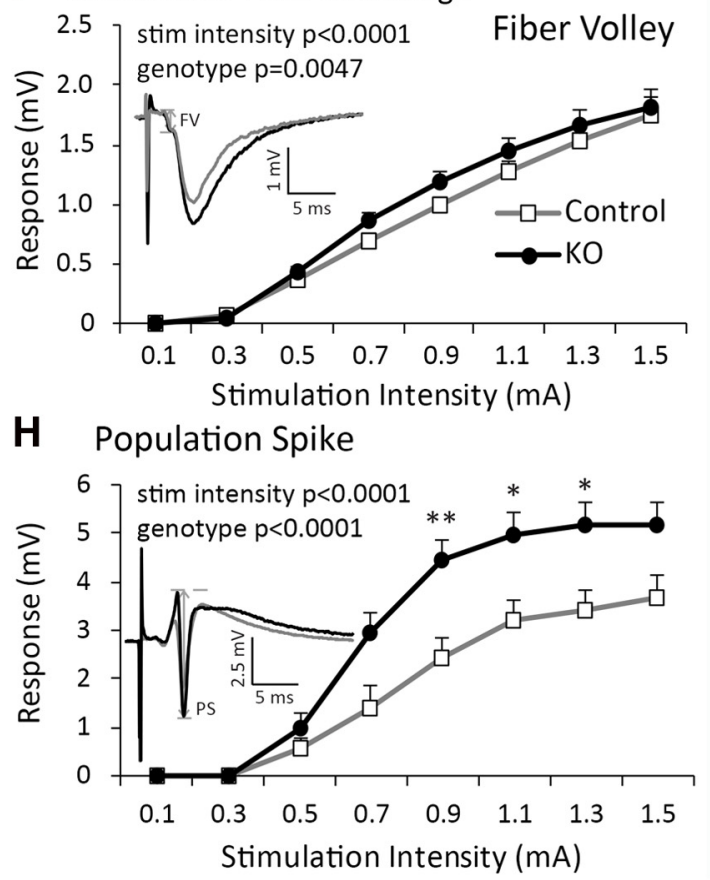

Figure 1. Postnatal deletion of astrocytic ephrin-B1 results in enhanced excitation of CA1 hippocampal neurons. $A$, Timeline of tamoxifen injection; $0.5 \mathrm{mg}$ of tamoxifen was intraperitoneally injected at P14 for 5 d; experiments were performed at P28, 14 days after initial injection. Three transgenic mice groups were used in this study: (1) ERT2-Cre ${ }^{\text {GFAP }}$ (Control) and ERT2Cre $^{\text {GFAP }}$ ephrin-B1 ${ }^{\text {flox/y }}$ (KO); (2) tdTomato- ERT2-Cre ${ }^{\text {GFA }}$ (Control) and tdTomato-ERT2-Cre ${ }^{\text {GFAP }}$ ephrin-B1 ${ }^{\text {flox/y }}$ (KO); and (3) Thy1-GFP- ERT2-Cre ${ }^{\text {GFAP }}$ (Control) and tdTomato-ERT2-Cre ${ }^{\text {GFAP }}$ ephrin$B 1^{\text {flox/y }}(K O)$. B, Schematic representation of mouse hippocampus with cornu ammonis (CA), DG, and hippocampal layers: SO, SP, SR, and SLM. Outlined box represents areas analyzed in these studies. C, D, Maximum projection confocal images represent astrocytes expressing tdTomato (red) and ephrin-B1 immunoreactivity (green) with DAPI (blue) in the (A1 hippocampus of Group 2 control and KO mice. Scale bar, $200 \mu \mathrm{m}$. High-magnification images represent examples of ephrin-B1 immunoreactivity in astrocytes (asterisk), and cell body (arrowhead) or dendrites (arrow) of CA1 neurons. Scale bar, $20 \mu \mathrm{m}$. $\boldsymbol{E}$, Ephrin-B1 immunoreactivity was significantly reduced in the astrocytes of CA1 hippocampus in K0 mice compared with control mice (left; $n=8-10, t$ test, ${ }^{*} p<0.05$; Extended Data Fig. 1-1). Ephrin-B1 immunoreactivity was also significantly reduced in the cell body of CA1 neurons (middle; $n=8-12, t$ test, $p<0.0001$; Extended Data Fig. 1-1) but was upregulated in proximal dendrites of CA1 neurons (right; $t$ test, $n=15-18, p<0.0001$; Extended Data Fig. 1-1). $\boldsymbol{F}-\boldsymbol{H}$, I/0 curves of CA1 neuronal FV amplitude (F), fEPSP slope (G), and PS amplitude $(\boldsymbol{H})$ as a function of increasing stimulation intensity of Schaffer collaterals in hippocampal slices from Group 1 control and K0 mice. Deletion of astrocytic ephrin-B1 resulted in increased fEPSP slope and PS amplitude following stimulation of Schaffer collaterals ( $n=6-9$ mice; two-way ANOVA followed by Bonferroni post-test; Extended Data Figs. 1-2, 1-3). Graphs represent mean. Error bars indicate SEM. ${ }^{*} p<0.05,{ }^{* *} p<0.01,{ }^{* * * *} p<0.0001$. 
$7.56 \times 10^{12} \mathrm{VPs} / \mathrm{ml}$ ) and control AAV-tdTomato VP (final concentration $4.46 \times 1012 \mathrm{VPs} / \mathrm{ml}$ ) were both obtained from UPenn Vector Core (http://www.med.upenn.edu/gtp/vectorcore) and processed as previously described (Koeppen et al., 2018) with modifications. VPs were concentrated with Amicon ultra-0.5 centrifugal filter (UFC505024, Sigma Millipore), which was pretreated with $0.1 \%$ Pluronic F-68 nonionic surfactant (24040032, Thermo Fisher Scientific). Mice were anesthetized with intraperitoneal injections of ketamine/xylazine mix $(80 \mathrm{mg} / \mathrm{kg}$ ketamine and $10 \mathrm{mg} / \mathrm{kg}$ xylazine). To ensure for adequate anesthesia, paw pad pinch test, respiratory rhythm, righting reflex, and/or loss of corneal reflex were assessed. P14 B6/C57 mice (RRID:IMSR_JAX: 000664) received craniotomies ( $1 \mathrm{~mm}$ in diameter), and VPs were stereotaxic injected into the dorsal hippocampus $(1.8 \mathrm{~mm}$ posterior to bregma, $1.1 \mathrm{~mm}$ lateral to midline, and $1.3 \mathrm{~mm}$ from the pial surface; see Fig. $3 A$ ). Control mice received a single injection of $1 \mu \mathrm{l}$ of $1.16 \times 10^{13}$ $\mathrm{VP} / \mathrm{ml}$ AAV-tdTomato, and experimental animals received a single injection of $1 \mu \mathrm{l}$ of $3.78 \times 10^{13} \mathrm{VP} / \mathrm{ml} \mathrm{AAV}$-ephrin-B1. After surgery, mice received $0.3 \mathrm{ml}$ of buprenorphine by subcutaneous injection every $8 \mathrm{~h}$ for $48 \mathrm{~h}$, as needed for pain. Animals were allowed to recover for $14 \mathrm{~d}$ before analysis. P28 mice were subjected to immunohistochemistry and whole-cell electrophysiology experiments. There was a significant increase in ephrin-B1 immunoreactivity in CA1 hippocampus on the injected ipsilateral side $(\mathrm{OE})$ compared with noninjected contralateral control side (see Fig. $3 B-D$ ).

Immunohistochemistry. Immunohistochemistry procedures were performed as described previously (Koeppen et al., 2018). Briefly, animals were anesthetized with isoflurane and transcardially perfused with $0.9 \% \mathrm{NaCl}$ followed by fixation with $4 \%$ PFA in $0.1 \mathrm{M} \mathrm{PBS}, \mathrm{pH} 7.4$. Brains were postfixed overnight with $4 \%$ PFA in $0.1 \mathrm{M}$ PBS, and $100 \mu \mathrm{m}$ coronal brain sections were obtained with a vibratome. Excitatory presynaptic boutons were labeled by immunostaining against vGlut1 using rabbit anti-vGlut1 antibody $(0.25 \mathrm{mg} / \mathrm{ml}$, Invitrogen, 482400, RRID:AB 2533843), and excitatory postsynaptic sites were identified with mouse anti-PSD95 antibody $(1.65 \mu \mathrm{g} / \mathrm{ml}$, Invitrogen, MA1-045, RRID:AB_ 325399). PV-positive cells were identified with mouse anti-PV antibody ( $2 \mu \mathrm{g} / \mathrm{ml}$, Sigma Millipore, P3088, RRID:AB_477329). Inhibitory presynaptic sites were detected by immunolabeling against vesicular GABA transporter (vGAT) using rabbit anti-vGAT antibody (1:100, Synaptic Systems, 131002, RRID:AB_887871). Inhibitory postsynaptic sites were detected by immunolabeling against gephyrin using mouse antigephyrin antibody (1:500, Synaptic Systems, 147111, RRID:AB_887719). Astrocytes were identified by immunolabeling against GFAP using mouse anti-GFAP antibody (1:500, Sigma Millipore, G3893, RRID:AB_ 477010), and ephrin-B1 immunoreactivity was detected by immunostaining with goat anti-ephrin-B1 antibody $(20 \mu \mathrm{g} / \mathrm{ml}$, R\&D Systems, AF473, RRID:AB_2293419). Secondary antibodies used were as follows: AlexaFluor-488-conjugated donkey anti-goat $\operatorname{IgG}(4 \mathrm{mg} / \mathrm{ml}$, Invitrogen, A-11055, RRID:AB_2534102), AlexaFluor-488-conjugated donkey antirabbit IgG (4 mg/ml, Invitrogen, A-21 206, RRID:AB_141708), AlexaFluor-594-conjugated donkey anti-mouse $\operatorname{IgG}(4 \mathrm{mg} / \mathrm{ml}$, Invitrogen, A-21 203, RRID:AB_141633), AlexaFluor-594-conjugated donkey antirabbit IgG (4 mg/ml, Invitrogen, AB150076, RRID:AB_2782993), and AlexaFluor-647-conjugated donkey anti-mouse IgG $(4 \mathrm{mg} / \mathrm{ml}$, Invitrogen, RRID:AB_2340863). Sections were mounted on slides with Vectashield mounting medium containing DAPI (Vector Laboratories, catalog \# H-1200, RRID:AB_2336790).

Confocal imaging and analysis. Confocal images of coronal brain slices containing stratum oriens (SO), stratum pyramidale (SP), stratum radiatum (SR), and stratum lacunosum-moleculare (SLM) layers in the CA1 hippocampus were taken with an SP5 confocal laser-scanning microscope (Leica Microsystems) as previously described (Koeppen et al., 2018). Briefly, high-resolution optical sections $(1024 \times 1024$ pixel format) were captured with a $40 \times$ water-immersion and $1 \times$ zoom at $1 \mu \mathrm{m}$ step intervals to assess ephrin-B1 immunoreactivity. Confocal images of dendritic spines and synaptic puncta were taken using a $63 \times$ objective (1.2 NA), and $1 \times$ zoom at high resolution $(1024 \times 1024$ pixel format $)$ with a $0.5 \mu \mathrm{m}$ intervals. All images were acquired under identical conditions and processed for analysis as follows: (1) For analysis of overall ephrin-B1 immunoreactivity, SO, SP, and SR layers of the CA1 hippocampus were analyzed per each brain slice from at least 3 animals per group. Each $z$ stack was collapsed into a single image by projection, converted to a tiff file, encoded for blind analysis, and analyzed using ImageJ software (RRID:nif-0000-30467). Each image was thresholdadjusted using default auto-threshold and then converted into a binary image. Selection tool was used to outline SO, SP, and SR layers of CA1 hippocampus based on DAPI stain, saved in the ROI manager, and used to measure integrated density, area, and the mean intensity. (2) For analysis of ephrin-B1 immunoreactivity in astrocytes, astrocytes were visualized with tdTomato and GFAP immunoreactivity. Cell areas were outlined using selection tool, then cell area, integrated fluorescent intensity, and mean intensity were measured for each astrocyte (100-200 astrocytes, $z$ stacks of at least 10 optical images, 3 mice per group, 2 or 3 brain slices per mouse). (3) For analysis of ephrin-B1 immunoreactivity in CA1 neurons, cell bodies in SP and their proximal dendrites in SR were randomly selected and outlined using selection tool. These ROIs were saved in the ROI manager and used to measure area and perform analysis of integrated fluorescent intensity and mean intensity. (4) For the analysis of vGlut1, PSD95, and PV immunolabeling, each image in the series was threshold-adjusted to identical levels (0-160 intensity) and puncta numbers $\left(0.5-10 \mu \mathrm{m}^{2}\right)$ were collected using ImageJ software. Three adjacent areas from SR and SLM were analyzed per each hippocampus from 4 animals per group. (5) For the analysis of gephyrin and vGAT-immunoreactive puncta, cell bodies and dendrites of GFPexpressing CA1 neurons were randomly selected for the analysis. Each $z$ stack was collapsed into a single image by projection, converted to a tiff file, encoded for blind analysis, and analyzed using ImageJ software (RRID:nif-0000-30467). Each image was threshold-adjusted using default autothreshold and then converted into a binary image. The watershed function was applied to each image to separate overlapping puncta. A selection tool was used to define ROIs around CA1 neuronal cell bodies in SP, primary and secondary dendrites in SR, and dendrites in SO layers. These ROIs were saved in the ROI manager and used to measure area and to perform puncta $\left(0.5-10 \mu \mathrm{m}^{2}\right)$ analysis using particle analysis tool. Colocalization of vGlut1/PSD95, vGlut1/PV, gephyrin/ vGAT was analyzed using ImageJ plugin for colocalization (https:// imagej.nih.gov/ij/plugins/colocalization.html). Statistical analysis was performed with two-way ANOVA followed by Tukey post hoc analysis or unpaired $t$ test using GraphPad Prism 7 software (RRID:SCR 002798). Data represent mean \pm SEM.

Dendritic spine analysis. Dendritic spines were identified with GFP in Thy1-GFP-ERT2-Cre ${ }^{G F A P}$ ephrin-B1 $1^{\text {flox/y }}(\mathrm{KO})$ or Thy1-GFP-ERT2Cre ${ }^{\text {GFAP }}$ (Control) male mice (Group 3) expressing GFP in hippocampal pyramidal neurons as previously described (Koeppen et al., 2018). Briefly, animals were anesthetized with isoflurane and transcardially perfused initially with $0.9 \% \mathrm{NaCl}$, followed by fixation with $4 \%$ PFA in $0.1 \mathrm{~m}$ PBS, pH 7.4. Brains were postfixed for $2 \mathrm{~h}$ in $4 \%$ PFA in $0.1 \mathrm{M}$ PBS, and $100 \mu \mathrm{m}$ coronal sections were obtained with a vibratome. CA1 hippocampal neurons were imaged using an SP5 confocal microscope (Leica Microsystems). Five GFP-expressing neurons were randomly selected per animal, and dendrites were imaged using a $63 \times$ oil-immersion objective (1.2 NA) and $1 \times$ zoom. Three-dimensional fluorescent images were created by the projection of each $z$ stack containing 50 high-resolution optical serial sections $(1024 \times 1024$ pixel format $)$ taken at $0.5 \mu \mathrm{m}$ intervals in the $\mathrm{X}-\mathrm{Y}$ plane. Quantifications of the spine density (spines per $10 \mu \mathrm{m}$ dendrite), lengths $(\mu \mathrm{m})$, and volumes $\left(\mu \mathrm{m}^{3}\right)$ were conducted using Neurolucida 360 software (MicroBrightField RRID: SCR_001775). Statistical analysis was performed with two-way ANOVA followed by Tukey post hoc analysis or unpaired $t$ test analysis using GraphPad Prism 7 software (GraphPad Prism, RRID:SCR_002798). Data represent mean \pm SEM.

Synaptosome isolation and Western blot analysis. Isolation of hippocampal synaptosomes was performed as previously described with modifications (Hollingsworth et al., 1985; Koeppen et al., 2018). Briefly, hippocampal tissues from P28 control or KO mice (Group 1) were homogenized in $1 \mathrm{ml}$ synaptosome buffer (in mM as follows: $124 \mathrm{NaCl}, 3.2$ $\mathrm{KCl}, 1.06 \mathrm{KH}_{2} \mathrm{PO}_{4}, 26 \mathrm{NaHCO}_{3}, 1.3 \mathrm{MgCl}_{2}, 2.5 \mathrm{CaCl}_{2}, 10$ glucose, 20 HEPES). Homogenates were first filtered through a $100 \mu \mathrm{m}$ nylon net filter (NY1H02500, Millipore) and then through a $5 \mu \mathrm{m}$ nylon syringe 
filter (SF15156, Tisch International). Homogenate flow-through was collected and synaptosomes were spun down at $10,000 \times g, 4^{\circ} \mathrm{C}$, for $30 \mathrm{~min}$. Synaptosomes were resuspended in $800 \mu \mathrm{l}$ synaptosome buffer. To confirm synaptosome enrichment, levels of synapsin-1 and PSD95 were analyzed in tissue homogenates and synaptosome fractions with Western blot analysis (see Extended Data Fig. 4-1). Isolated hippocampal synaptosome samples were centrifuged at $10,000 \times \mathrm{g}, 4^{\circ} \mathrm{C}$, for $30 \mathrm{~min}$, and pellets were resuspended in lysis buffer $(50 \mathrm{~mm}$ Tris, $100 \mathrm{~mm} \mathrm{NaCl}, 2 \%$ Triton X-100, $10 \mathrm{~mm}$ EDTA) containing $2 \%$ protease inhibitor cocktail (P8340, Sigma Millipore) and incubated for $2 \mathrm{~h}$ at $4^{\circ} \mathrm{C}$. Samples were added to $2 \times$ Laemmli Buffer (S3401, Sigma Millipore) and run on an 8\%-16\% Tris-glycine Gel (EC6045BOX, Invitrogen). Protein samples were transferred onto a nitrocellulose blotting membrane (10600007, GE Healthcare). Blots were blocked with 5\% milk in TBS (10 mм Tris, 150 $\mathrm{mm} \mathrm{NaCl}$, pH 8.0), followed by immunostaining with mouse anti-PSD95 $(1.65 \mu \mathrm{g} / \mathrm{ml}$, Invitrogen, MA1-045, RRID:AB_325399), rabbit antiGluA1 (1:100, Millipore, AB1504, RRID:AB_2113602), rabbit antiGluA2/3 $(0.1 \mu \mathrm{g} / \mathrm{ml}$, Millipore, AB1506, RRID:AB_90710), rabbit anti-synapsin-1 (0.2 $\mu \mathrm{g} / \mathrm{ml}$, Millipore, AB1543P, RRID:AB_90757), or mouse anti-GAPDH $(0.2 \mu \mathrm{g} / \mathrm{ml}$, Thermo Fisher Scientific, 39-8600, RRID:AB_2533438) antibodies in $0.1 \%$ Tween $20 / \mathrm{TBS}$ at $4^{\circ} \mathrm{C}$ for $16 \mathrm{~h}$. Secondary antibodies used were HRP-conjugated donkey anti-mouse IgG (Jackson ImmunoResearch Laboratories, 715-035-150, RRID:AB_ 2340770) or HRP-conjugated goat anti-rabbit IgG (Jackson ImmunoResearch Laboratories, 111-035-003, RRID:AB_2313567). Blots were incubated in ECL 2 Western Blotting Substrate (80196, Pierce), and a signal was collected with CL-XPosure film (34090, Pierce). Band density was analyzed by measuring band and background intensity using Adobe Photoshop CS5.1 software (RRID:SCR_014199). Statistical analysis was performed with unpaired $t$ test using GraphPad Prism 7 software (RRID: SCR_002798). Data represent mean \pm SEM.

Extracellular field recordings. P28 mice (Group 1) were used for electrophysiological experiments, 2 weeks after the first tamoxifen injection. Animals were deeply anesthetized with isoflurane and decapitated. The brains were rapidly removed and immersed in ice-cold ACSF with high $\mathrm{Mg}^{2+}$ and sucrose concentration containing the following (in $\mathrm{mM}$ ): 3.5 $\mathrm{KCl}, 1.25 \mathrm{NaH}_{2} \mathrm{PO}_{4}, 20 \mathrm{D}(+)$-glucose, 185 sucrose, $26 \mathrm{NaHCO}_{3}, 10$ $\mathrm{MgCl}_{2}$, and $0.50 \mathrm{CaCl}_{2}, \mathrm{pH}$ of 7.4 , and saturated with $95 \% \mathrm{O}_{2} / 5 \% \mathrm{CO}_{2}$. Transverse $350 \mu \mathrm{m}$ hippocampal slices were prepared by using a vibrating blade microtome (LeicaVT1000s, Leica Microsystems) in ice-cold slicing solution bubbled with $95 \% \mathrm{O}_{2} / 5 \% \mathrm{CO}_{2}$. Slices were then transferred into a holding chamber containing oxygenated ACSF (in mм as follows: $124 \mathrm{NaCl}, 3.5 \mathrm{KCl}, 1.25 \mathrm{NaH}_{2} \mathrm{PO}_{4}, 10 \mathrm{D}(+)$-glucose, 26 $\mathrm{NaHCO}_{3}, 2 \mathrm{MgCl}_{2}$, and $2 \mathrm{CaCl}_{2}, \mathrm{pH} 7.4$ ) and incubated for $1 \mathrm{~h}$ at $33^{\circ} \mathrm{C}$, then kept at room temperature. For recordings, slices were transferred to a recording chamber and continuously perfused with oxygenated ACSF at a flow rate of $1 \mathrm{ml} / \mathrm{min}$ at $33^{3} \mathrm{C}$. Slices were equilibrated in recording chamber for $10 \mathrm{~min}$ to reach a stable baseline response before running experimental protocols. Glass microelectrodes were pulled with a Sutter P-97 micropipette puller (Sutter Instrument; RRID:SCR_016842) with a tip resistance of 1-3 $\mathrm{M} \Omega$ and filled with $3 \mathrm{M} \mathrm{NaCl}$. Glass microelectrodes were positioned in the SP and SR areas of CA1 hippocampus for extracellular recording. Synaptic responses were evoked by stimulating Schaffer collaterals using a bipolar tungsten electrode (WPI), $\sim 200 \mu \mathrm{m}$ away from the recording electrodes. Potentials were amplified (Axoclamp2B, Molecular Devices), digitized at a sampling rate of $10 \mathrm{kHz}$, and analyzed offline using pClamp 10.7 software (Molecular Devices; RRID:SCR_ 011323). All electrophysiological responses were digitally filtered at $1 \mathrm{kHz}$ low-pass filter to improve signal-to-noise ratio.

Dendritic potentials typically consisted of a small presynaptic fiber volley (FV) followed by a negative fEPSP. The amplitude of the FV reflects the depolarization of the presynaptic terminals and was quantified by measuring the amplitude of first negative waveform. The fEPSP slope reflects the magnitude of the postsynaptic dendritic depolarization and was quantified by measuring the $20 \%-80 \%$ slope of the second negative waveform. Postsynaptic neuronal firing is represented by the amplitude of the population spike (PS), which was calculated as the voltage difference between first positive peak and the most negative peak of the trace.

Input-output (I/O) curves were generated to examine basal synaptic transmission by incrementally increasing stimulation intensity, beginning at $0.10 \mathrm{~mA}$ and increasing stimulation by $0.10 \mathrm{~mA}$ until maximal somatic PS amplitude was reached. Maximal PS amplitude was regarded as maximal neuronal output. Maximal fEPSP slope and PS response along with $30 \%-50 \%$ of maximal fEPSP slope and PS were determined.

For electrophysiological data, two-way ANOVA was used followed by Bonferroni test to evaluate the effects of astrocytic ephrin-B1 deletion on the $\mathrm{I} / \mathrm{O}$ curves. In all electrophysiological recordings, the data represent mean \pm SEM.

Whole-cell patch-clamp recordings. Brain slice preparation for whole-cell patch clamp was the same as above; briefly, brains were rapidly removed and immersed in ice-cold "slushy" ACSF with high $\mathrm{Mg}^{2+}$ and sucrose concentration containing the following (in mM): $87 \mathrm{NaCl}$, 75 sucrose, $2.5 \mathrm{KCl}, 0.5 \mathrm{CaCl}_{2}, 7 \mathrm{MgCl}_{2}, 1.25 \mathrm{NaH}_{2} \mathrm{PO}_{4}, 25 \mathrm{NaHCO}_{3}, 10$ glucose, 1.3 ascorbic, acid, 0.1 kynurenic acid, 2.0 pyruvate, and 3.5 MOPS, $\mathrm{pH} 7.4$, and saturated with $95 \% \mathrm{O}_{2} / 5 \% \mathrm{CO}_{2}$. Transverse hippocampal slices $(350 \mu \mathrm{m})$ were prepared by using a vibrating blade microtome (Campden 5100mz-Plus, Campden Instruments) in high $\mathrm{Mg}^{2+}$ / sucrose ACSF solution bubbled with $95 \% \mathrm{O}_{2} / 5 \% \mathrm{CO}_{2}$. Slices were then incubated in a holding chamber containing oxygenated high $\mathrm{Mg}^{2+} / \mathrm{su}_{-}$ crose ACSF for $30 \mathrm{~min}$ at room temperature and then transferred into ACSF (in mm as follows: $125 \mathrm{NaCl}, 2.5 \mathrm{KCl}, 2.5 \mathrm{CaCl}_{2}, 1.3 \mathrm{MgCl}_{2}, 1.25$ $\mathrm{NaH}_{2} \mathrm{PO}_{4}, 26 \mathrm{NaHCO}_{3}, 15$ glucose, $3.5 \mathrm{MOPS}, \mathrm{pH} 7.4$ ) for an additional $30 \mathrm{~min}$ at room temperature. Slices were transferred to a recording chamber and continually perfused with oxygenated ACSF at a flow rate of $1 \mathrm{ml} / \mathrm{min}$ at $33^{3} \mathrm{C}$.

Whole-cell patch experiments were conducted blind, as described by Castaneda-Castellanos et al. (2006). Electrical stimuli $(0.1 \mathrm{~Hz})$ were delivered through a bipolar, Teflon-coated tungsten electrode (FHC) placed in the SR area of CA1 hippocampus to stimulate Schaffer collaterals, $\sim 200 \mu \mathrm{m}$ away from recording electrode, and CA1 hippocampal pyramidal neurons were voltage-clamped. Tight-seal whole-cell recordings were obtained using pipettes made from borosilicate glass capillaries pulled on a Narishige PC-10 vertical micropipette puller (Narishige). Pipette resistance ranged from 3 to $4 \mathrm{M} \Omega$ and filled with an internal solution containing the following (in $\mathrm{mm}$ ): $130 \mathrm{CsOH}, 130 \mathrm{D}$-gluconic acid, 0.2 EGTA, $2 \mathrm{MgCl}_{2}$, $6 \mathrm{CsCl}$, 10 HEPES, 2.5 ATP-Na, 0.5 GTP-Na, 10 phosphocreatine, and $0.1 \%$ biocytin for cellular postlabeling, $\mathrm{pH}$ adjusted to 7.2-7.3 with $\mathrm{CsOH}$, osmolarity adjusted to 300-305 mOsm with ATP-Na. CA1 neurons were voltage-clamped at either $-70 \mathrm{mV}$ to measure AMPAR-evoked responses or $40 \mathrm{mV}$ to measure NMDARevoked responses. Amplitudes of evoked AMPAR-mediated responses were measured at peak response following stimulus artifact, and amplitudes of evoked NMDAR-mediated responses were measured $60 \mathrm{~ms}$ following stimulus artifact to isolate NMDAR-only mediated responses; $1 \mu \mathrm{M}$ TTX was added to isolate mEPSC responses. All EPSCs were recorded in the presence of $50 \mu \mathrm{M}$ picrotoxin, a GABA $\mathrm{A}_{\mathrm{A}}$ receptor antagonist, to block $\mathrm{GABA}_{\mathrm{A}}$-mediated currents. To measure IPSCs, electrical stimulation was delivered in the SR region to stimulation inhibitory neurons (in close proximity, $\sim 200 \mu \mathrm{m}$ away from recording electrode) and CA1 hippocampal pyramidal neurons were voltage-clamped and recorded at $0 \mathrm{mV}$ in the presence of $10 \mu \mathrm{M}$ NBQX, an AMPAR antagonist, and $50 \mu \mathrm{M}$ D-AP5, an NMDAR antagonist. EPSCs and IPSCs were recorded using an EPC-9 amplifier (HEKA Elektronik), filtered at $1 \mathrm{kHz}$, digitized at $10 \mathrm{kHz}$, and stored on a personal computer using pClamp 10.7 software (Molecular Devices) to run analysis. The series resistance was $<25 \mathrm{M} \Omega$ and was compensated. Both series and input resistances were monitored throughout the experiment by delivering $5 \mathrm{mV}$ voltage steps. If the series resistance changed $>20 \%$ during the course of an experiment, the data were discarded. AMPAR- and NMDAR-mediated evoked EPSCs, evoked IPSCs, mEPSCs, and mIPSCs were analyzed by Clampfit 10.7 software (Molecular Devices) and customized MATLAB script. Miniature events with the amplitude of $\geq 5 \mathrm{pA}$ were included in the analysis. To test the effects of ephrin-B1 KO in astrocytes, Group 1 $\mathrm{KO}$ male mice and their control littermates were used. To test the effects of ephrin-B1 OE, recordings from AAV-ephrin-B1-injected ipsilateral side were included in the OE group. As no significant differences were observed between recordings from contralateral noninjected side and AAV-tdTomato-injected hippocampi, both were combined in the control group. All averaged data were presented as mean \pm SEM. Statistical 
significance was determined by unpaired Student's $t$ test using Prism 7 software (GraphPad Software).

Social novelty test. Sociability and social memory were studied in $\mathrm{KO}$ male mice and their control littermates (Group 1) using a three-chamber test as described previously (Kaidanovich-Beilin et al., 2011). Briefly, a rectangular box contained three adjacent chambers $19 \times 45 \mathrm{~cm}$ each, with $30-\mathrm{cm}$-high walls and a bottom constructed from clear Plexiglas. The three chambers were separated by dividing walls, which were made from clear Plexiglas with openings between the middle chamber and each side chamber. Removable doors over these openings permitted chamber isolation or free access to all chambers. All testing was done in a brightly lit room (650lux), between 9:00 A.M. and 2:00 P.M. Before testing, mice were housed in a room with a $12 \mathrm{~h}$ light/dark cycle with ad libitum access to food and water. The cages were transferred to the behavioral room $30 \mathrm{~min}$ before the first trial began for habituation. The test mouse was placed in the central chamber with no access to the left and right chambers and allowed to habituate to the test chamber for 5 min before testing began. Session 1 measured sociability; in Session 1, another mouse (Stranger 1) was placed in a wire cup-like container in one of the side chambers. The opposite side had an empty cup of the same design. The doors between the chambers were removed, and the test mouse was allowed to explore all three chambers freely for $10 \mathrm{~min}$, while being digitally recorded from above. The following parameters were monitored: the duration of direct contact between the test mouse and either the stranger mouse or empty cup and the duration of time spent in each chamber. Session 2 measured social memory; in Session 2, a new mouse (Stranger 2) was placed in the empty wire cup in the second side chamber. Stranger 1, a now familiar mouse, remained in the first side chamber. The test mouse was allowed to freely explore all three chambers for another $10 \mathrm{~min}$, while being recorded, and the same parameters were monitored. Placement of Stranger 1 in the left or right side of the chamber was randomly altered between trials. The floor of the chamber was cleaned with $2 \%-3 \%$ acetic acid, $70 \%$ ethanol, and water between tests to eliminate odor trails. Assessments of the digital recordings were done using TopScan Lite software (Clever Sys). To measure changes in social preference and social memory, percent time spent in each chamber was calculated in each test. Further, a social preference index $\left(\frac{\text { time in } S 1 \text { chamber }}{\text { time in } S 1 \text { chamber }+ \text { time in empty chamber }}\right)$ and social novelty index $\left(\frac{\text { time in } S 2 \text { chamber }}{\text { time in } S 2 \text { chamber }+ \text { time in } S 1 \text { chamber }}\right)$ were calculated as described previously (Nygaard et al., 2019). For social preference index, values $<0.5$ indicate more time spent in the empty chamber, $>0.5$ indicate more time spent in the chamber containing Stranger 1, and 0.5 indicates equal amount of time in both chambers. For social novelty index, values $<0.5$ indicate more time spent in the chamber containing Stranger 1 or now familiar mouse, $>0.5$ indicate more time spent in the chamber containing Stranger 2 or new stranger mouse, and 0.5 indicates equal amount of time in both chambers. Statistical analysis was performed using two-way ANOVA followed by Tukey's post hoc test.

Open field behavior test. Locomotor activity and anxiety were evaluated using a standard open field exploration test as previously described with modifications (Lovelace et al., 2020). The apparatus consisted of a $42.5 \times 30 \mathrm{~cm}$ open field arena with 30 - $\mathrm{cm}$-high walls constructed from opaque acrylic sheets and a clear acrylic sheet for the bottom with a grid placed underneath it for scoring purposes. All testing was done in a brightly lit room (650lux), between 9:00 A.M. and 2:00 P.M. Before testing, mice were housed in a room with a $12 \mathrm{~h}$ light/dark cycle with ad libitum access to food and water. Mice were initially habituated to the testing room for at least $30 \mathrm{~min}$ before testing. During testing, animals were allowed to freely explore the open field for $10 \mathrm{~min}$. The floor of the chamber was cleaned with $2 \%-3 \%$ acetic acid, $70 \%$ ethanol, and water between tests to eliminate odor trails. Assessments of the digital recordings were done by blinded observers using TopScan Lite software (Clever Sys). A tendency to travel to the center and percent time spent in thigmotaxis were used as an indicator of anxiety (Yan et al., $2004,2005)$. Average velocity and total line crosses were measured to score locomotor activity. Statistical analysis was performed using Student's $t$ test.

\section{Results}

In the current study, we examined whether deletion or OE of ephrin-B1 in astrocytes during a developmental critical period affects the formation and maturation of synapses in the hippocampus. First, we evaluated E/I synaptic changes using both extracellular field recordings and whole-cell patch-clamp electrophysiology. Next, we assessed the number of dendritic spines and synapses on CA1 hippocampal neurons using immunohistochemistry. Finally, social behaviors, anxiety, and hyperactivity were evaluated in ephrin-B1 KO mice to examine functional significance of the astrocyte-specific deletion of ephrin-B1 during postnatal brain development.

\section{Ephrin-B1 loss in developing astrocytes enhances excitability of CA1 hippocampal neurons}

Previously, we have shown that astrocytic ephrin-B1 is involved in the maintenance of excitatory, but not inhibitory, synapses in the adult hippocampus (Koeppen et al., 2018; Nguyen et al., 2020). Ephrins and Eph receptors are identified as risk genes for the development of ASD in humans (Sanders et al., 2012). Mouse models of ASDs are also shown to have altered E/I balance as a result of aberrant E/I synapse development and plasticity, inhibitory neuron development, neuronal excitability, and glial cell dysfunction (Lee et al., 2017). Therefore, we assessed the effects of astrocyte-specific deletion or OE of ephrin-B1 in developing hippocampus during the critical developmental period when maturation of neuronal circuits occurs.

To achieve specific ephrin-B1 deletion in astrocytes, ERT2Cre ${ }^{G F A P}$ ephrin-B1 ${ }^{\text {flox/y }} \mathrm{KO}$ mice (Group 1) were generated and ERT2-Cre ${ }^{\text {GFAP }}$ mice were used as a control (Fig. $1 A$ ). To allow for tdTomato expression in astrocytes and analysis of ephrin-B1 levels in astrocytes, tdTomatoERT2-Cre ${ }^{\text {GFAP }}$ ephrin-B1 $1^{\text {flox/y }} \mathrm{KO}$ mice (Group 2) were generated and tdTomatoERT2-Cre ${ }^{G F A P}$ mice were used as controls (Fig. 1A). To achieve GFP expression in excitatory pyramidal neurons for analysis of dendritic spines and synapses, Thy1-GFP-ERT2-Cre ${ }^{\text {GFAP }}$ ephrin-B1 ${ }^{\text {flox/y }}$ KO (Group 3 ) and their corresponding Thy1-GFP-ERT2-Cre ${ }^{\text {GFAP }}$ control mice were generated (Fig. 1A). In all groups of mice, KO and their littermate control mice received tamoxifen at P14 intraperitoneally $(0.5 \mathrm{mg}$ in $5 \mathrm{mg} / \mathrm{ml}$ of 1:9 ethanol/sunflower seed oil solution) once a day for 5 consecutive days, and analysis was performed at P28 (Fig. 1A). To confirm specific ablation of ephrin-B1 in astrocytes, ephrin-B1 immunoreactivity was analyzed in the CA1 hippocampus (Fig. 1B) of mouse Group 1 (ERT2Cre ${ }^{G F A P}$ ephrin-B1 $1^{\text {flox/y }} \mathrm{KO}$ and their control littermates) and Group 2 (tdTomatoERT2-Cre ${ }^{\text {GFAP }}$ ephrin-B1 $1^{\text {flox/y }} \mathrm{KO}$ and their control littermates; Fig. 1C,D). Ephrin-B1 immunoreactivity was significantly reduced in hippocampal astrocytes of $\mathrm{KO}$ compared with control mice (Fig. 1C-E; Extended Data Fig. 1$1, t$ test, $\left.t_{(16)}=2.908 p<0.0103\right)$. We also observed changes in the distribution of ephrin-B1 immunoreactivity in CA1 neurons of $\mathrm{KO}$ mice (Fig. $1 C, D$ ) with a significant reduction in cell bodies (Fig. 1E; Extended Data Fig. $1-1 ; t$ test, $t_{(18)}=5.538$ $p<0.0001)$ and an increase in proximal dendrites compared with control mice (Fig. $1 E$; Extended Data Fig. $1-1$; $t$ test, $t_{(31)}=$ $5.326 p<0.0001)$.

To determine whether loss of astrocytic ephrin-B1 alters neuronal activity in the developing hippocampus, acute hippocampal slices were prepared for extracellular field recordings from Group $1 \mathrm{KO}$ male mice and their control counterparts at P28. Presynaptic FV amplitude, postsynaptic fEPSP slope, and somatic PS amplitude of neuronal responses were recorded in SR 
A

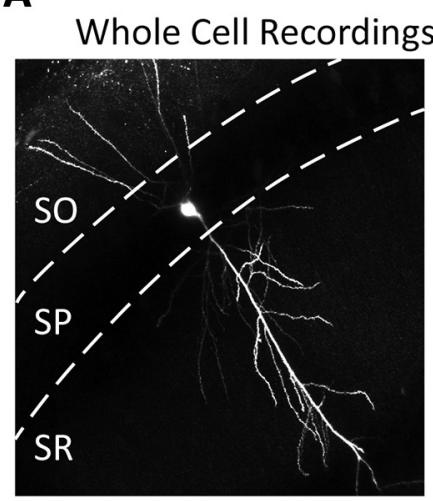

B

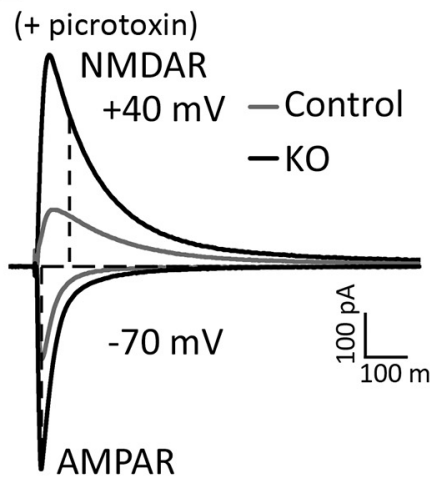

C

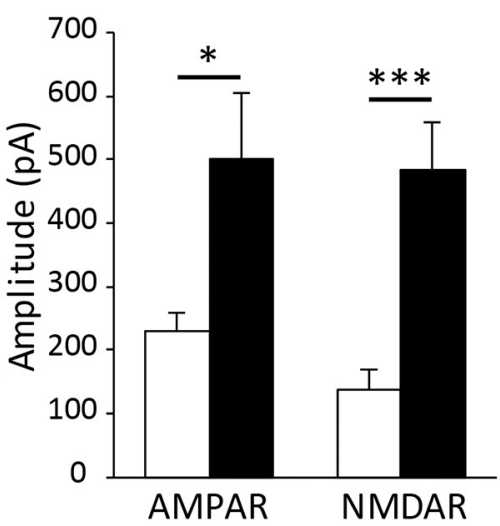

D

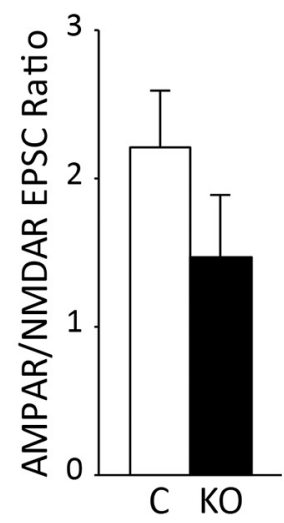

E $\operatorname{mEPSC}$ (+ picrotoxin, TTX)

Control

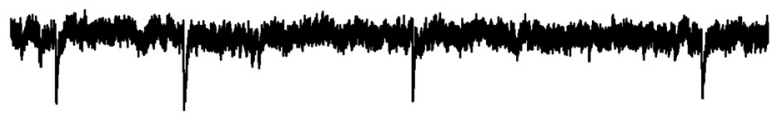

$\mathbf{F}$

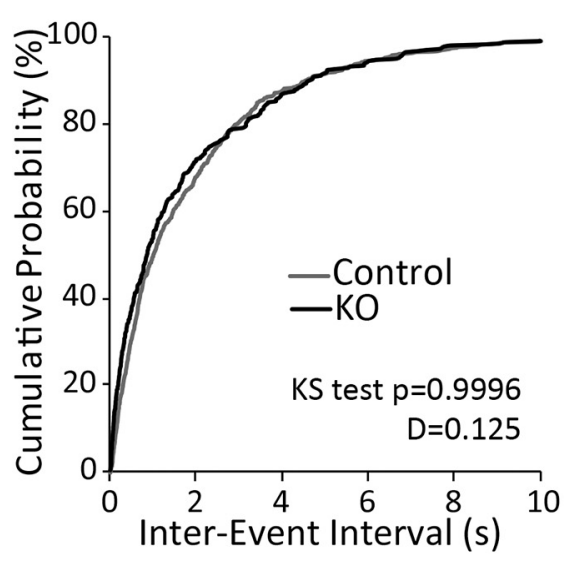

G

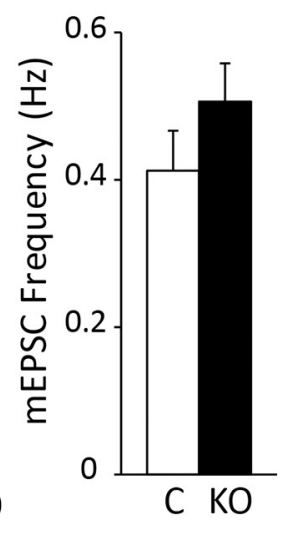

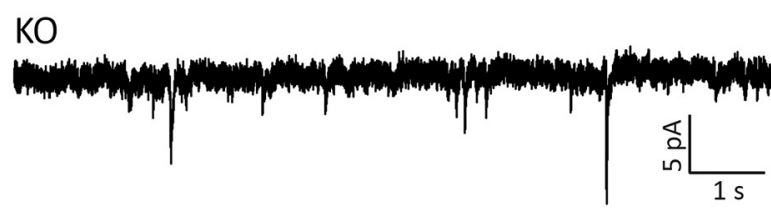

H

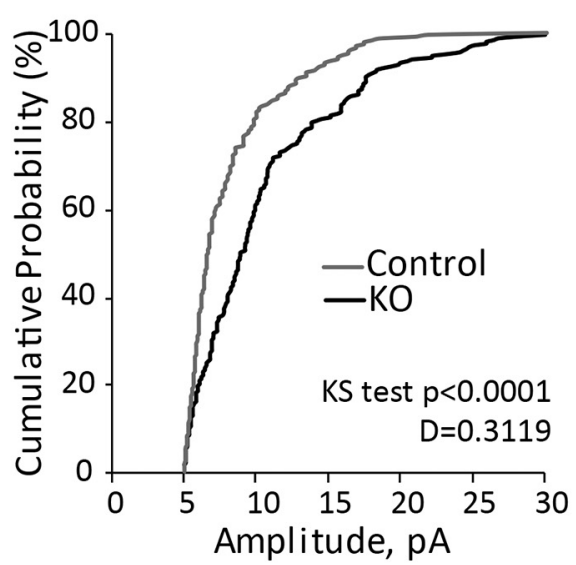

I

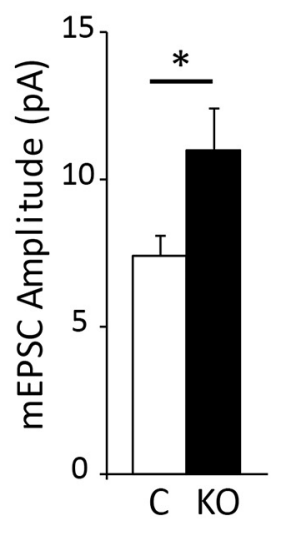

Figure 2. Loss of astrocytic ephrin-B1 during early postnatal development enhances both AMPAR- and NMDAR-mediated responses but not AMPAR/NMDAR EPSC ratio. A, Whole-cell recordings were performed by blind cell patching of pyramidal cells in the CA1 hippocampus (example of biocytin-filled neuron). $\boldsymbol{B}$, Representative traces of AMPAR- and NMDAR-evoked responses in control (gray) and KO (black) mice in the presence of picrotoxin to block GABAergic inhibition. $C$, $D$, Graphs represent amplitude and corresponding ratio of AMPAR- and NMDAR-mediated currents ( $n=10$ or 11 cells, 6 or 7 mice; Extended Data Fig. 2-1). Evoked AMPAR- and NMDAR-mediated currents were significantly increased; however, ratio was unchanged. $\boldsymbol{E}$, Representative traces of mEPSCS in P28 control and KO mice, recorded in the presence of TTX and picrotoxin ( $n=5$ mice). $\boldsymbol{F}$, The cumulative distribution of mEPSC interevent intervals shows no differences between control and KO mice. $G$, Average frequency of mEPSCs was not significantly different between control and K0 mice, indicating potentially no effect on presynaptic activity (Extended Data Fig. 2-1). $\boldsymbol{H}$, The cumulative distribution of mEPSC amplitude shows a significant rightward shift (higher mESPC amplitude across the distribution) for KO (black) mice compared with control (gray). I, Average amplitude of mEPSCs was higher in K0 compared with control mice (Extended Data Fig. 2-1). Graphs represent mean. Error bars indicate SEM. $t$ test: ${ }^{*} p<0.05$; ${ }^{* * *} p<0.001$.

and SP layers of CA1 hippocampus, and I/O curves were generated by incrementally increasing stimulation intensity of Schaffer collateral in CA3 hippocampus (Fig. $1 F-H$ ). Extracellular field recordings revealed that the loss of astrocytic ephrin-B1 increased the excitability of CA1 hippocampal neurons. Both FV response amplitude (Fig. 1F; Extended Data Figs. 1-2, 1-3; twoway ANOVA; stimulation intensity $F_{(14,540)}=89.41, p<0.0001$; genotype $\left.F_{(1,540)}=8.064, p=0.0047\right)$ and fEPSP slope were significantly higher in $\mathrm{KO}$ mice (Fig. $1 G$; Extended Data Figs. 1-2, 1-3; two-way ANOVA; stimulation intensity $F_{(14,386)}=41.58$, $p<0.0001$; genotype $\left.F_{(1,386)}=39.26, p<0.0001\right)$. PS response amplitude was also greatly enhanced in $\mathrm{KO}$ mice (Fig. $1 H$; Extended Data Figs. 1-2, 1-3; two-way ANOVA; stimulation intensity $F_{(14,510)}=42.41, p<0.0001$; genotype $F_{(1,510)}=64.18$, $p<0.0001)$.
These results demonstrate that hippocampal CA1 pyramidal neurons show enhanced excitability following astrocyte specific deletion of ephrin-B1 during early postnatal development, suggesting an alteration in $\mathrm{E} / \mathrm{I}$ balance.

Enhanced evoked excitatory postsynaptic AMPAR- and NMDAR-mediated responses and higher mEPSC amplitude are detected in CA1 neurons of astrocyte-specific ephrin-B1 KO mice

To determine the mechanism of enhanced hippocampal activity in astrocyte-specific ephrin-B1 KO mice, whole-cell voltageclamp electrophysiology was used to measure spontaneous and evoked excitatory responses from CA1 pyramidal neurons of P28 control and $\mathrm{KO}$ mice in the presence of $\mathrm{GABA}_{\mathrm{A}}$ receptor 
A Developmental period P14-28

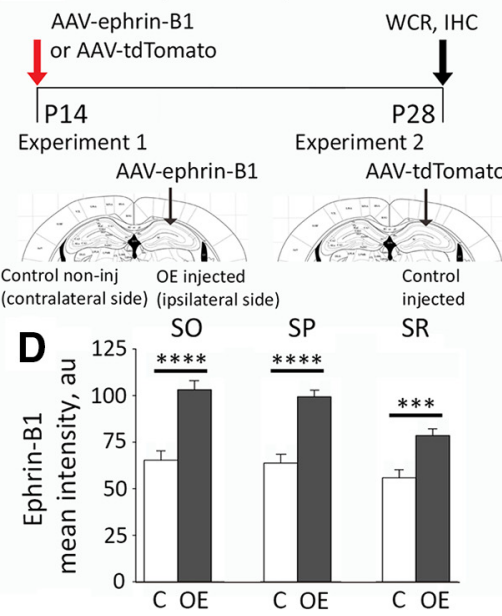

E Whole Cell Recordings (+ picrotoxin)

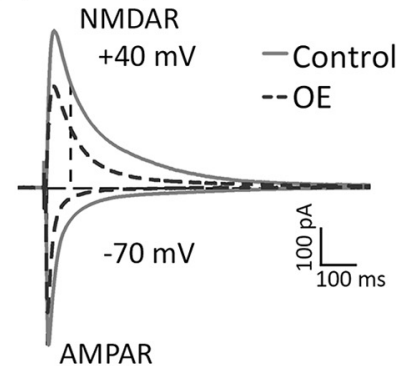

H mEPSC (+ picrotoxin, TTX) Control

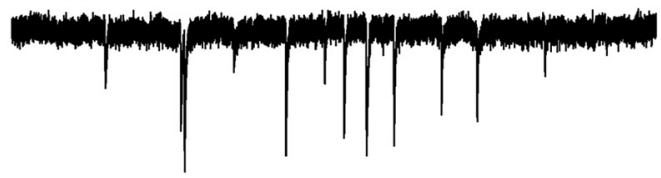

I
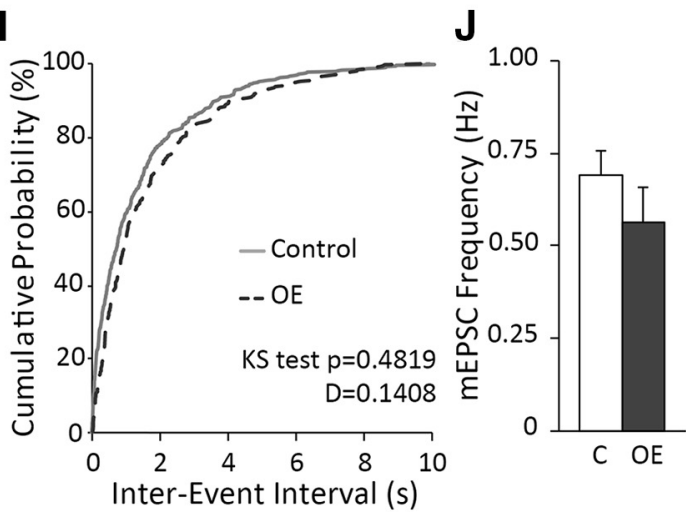

B Control (contralateral side)

ephrin-B1 GFAP DAPI

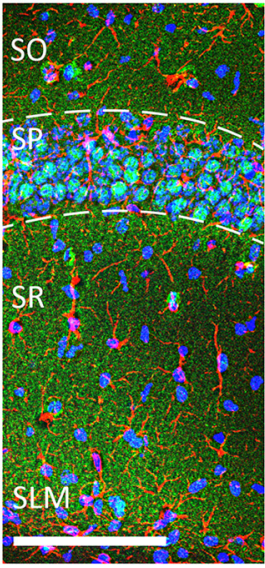

$\mathbf{F}$
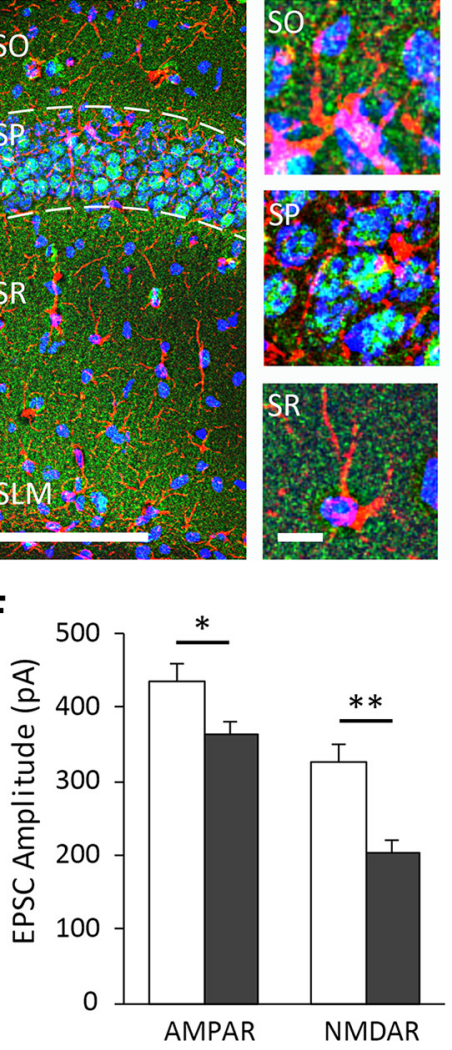

C OE (ipsilateral side)

ephrin-B1 GFAP DAPI
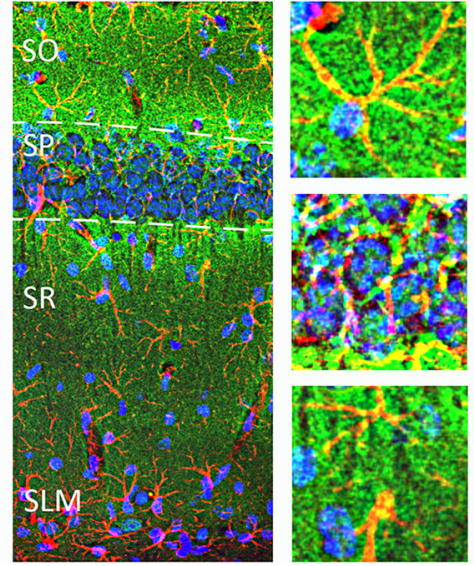

G

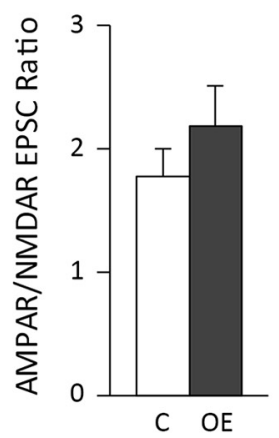

$\square$ Control $\square \mathrm{OE}$
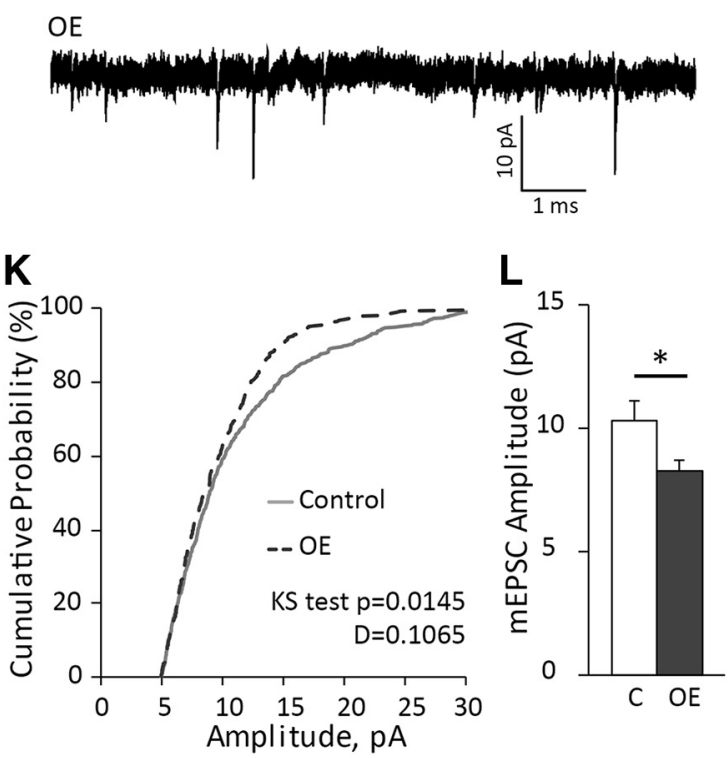

Figure 3. OE of astrocytic ephrin-B1 in the developing hippocampus reduced evoked AMPAR- and NMDAR-mediated responses. A, Timeline of the VP injection: AAV-ephrin-B1 or AAVtdTomato was stereotaxically injected unilaterally in the dorsal hippocampus at P14; experiments were performed at P28, 14 days after injection. $B, C$, Maximum projection confocal images represent GFAP (red) and ephrin-B1 (green) immunoreactivity with DAPI (blue) in the ipsilateral CA1 hippocampus injected with AAV-ephrin-B1 (OE) and contralateral noninjected CA1 hippocampus (Control). Scale bar, $200 \mu \mathrm{m}$. High-magnification images represent examples of ephrin-B1 immunoreactivity in astrocytes and neuronal cell bodies in SO, SP, and SR layers of CA1 hippocampus. Scale bar, $20 \mu \mathrm{m}$. D, Ephrin-B1 immunoreactivity was significantly increased in all layers of CA1 hippocampus in ipsilateral CA1 hippocampus injected with AAV-ephrin-B1 (OE) compared with contralateral noninjected CA1 hippocampus (Control) $\left(n=11-13, t\right.$ test, ${ }^{* * *} p<0.001$; ${ }^{* * *} p<0.0001$; Extended Data Fig. 3-1). E, For electrophysiology, control group included recordings from ipsilateral side of AAV-tdTomato-injected mice and contralateral side of AAV-ephrin-B1-injected mice. Overexpressing group (OE) included recordings from ipsilateral side of AAV-ephrin-B1-injected mice. Representative traces of AMPAR- and NMDAR-evoked responses in CA1 hippocampal neurons of control (gray) and OE (dotted dark gray) P28 hippocampus with whole-cell recording in the presence of picrotoxin to block GABAergic inhibition. $F, G$, Graphs represent amplitude and corresponding ratio in AMPAR- and NMDAR-mediated currents ( $n=11-14$ cells, 5 mice per group; Extended Data Fig. 3-2). Evoked AMPAR- and NMDAR-mediated currents were significantly decreased; however, ratio was unchanged. $\boldsymbol{H}$, Representative traces of mEPSCs in CA1 hippocampal neurons of P28 control and OE hippocampus; whole-cell recording was done in the presence of TTX and picrotoxin ( $n=11-14$ cells, 5 mice per group). $I$, The cumulative distribution of mEPSC interevent intervals in control (black) and OE (gray) groups. J, Average frequency of mEPSCs was not significantly different between control and OE groups, 
antagonist picrotoxin (Fig. 2). I/O curves were generated (not shown), and stimulation that induced maximum response was used to measure AMPAR- and NMDAR-mediated EPSCs. We observed a significant increase in both AMPAR- and NMDARmediated EPSCs in KO mice compared with control (Fig. 2C; Extended Data Fig. 2-1; AMPAR: $t$ test, $t_{(16)}=2.393, p=0.0293$; NMDAR: $t$ test, $\left.t_{(19)}=4.10, p=0.0006\right)$. Interestingly, AMPAR/ NMDAR ratio was not significantly different between control and $\mathrm{KO}$ mice (Fig. 2D; Extended Data Fig. $2-1 ; t_{(16)}=1.297$, $p=0.2130)$. There were also no differences in mEPSC frequencies between control and KO mice (Fig. 2F, G; Extended Data Fig. $2-1 ; t$ test, $\left.t_{(9)}=1.259, p=0.2398\right)$. In contrast, we observed a significant shift in cumulative probability distribution of mEPSC amplitude to a higher amplitude (Fig. $2 \mathrm{H}$; K-S test, $n=190$ and 160 for control and $\mathrm{KO}$ group, respectively, $p<0.0001, \mathrm{D}=$ 0.3119 ) and increased mEPSC average amplitude (Fig. 2I; Extended Data Fig. 2-1; $t$ test, $t_{(9)}=2.208, p=0.0273$ ). Our results suggest enhanced postsynaptic excitatory responses in CA1 neurons following astrocyte-specific deletion of ephrin-B1 during the P14-P28 developmental period.

\section{OE of astrocytic ephrin-B1 in hippocampus CA1 affects AMPAR- and NMDAR-mediated responses and mEPSC amplitude}

Next, we examined the effects of the OE of ephrin-B1 in hippocampal astrocytes during P14-P28 on excitatory activity in CA1 pyramidal neurons. To achieve OE of ephrin-B1 or control (C) tdTomato in hippocampal astrocytes, we used VPs containing ephrin-B1 cDNA (AAV-ephrin-B1) or tdTomato cDNA (AAVtdTomato). VPs were stereotaxic injected into the dorsal hippocampus (Fig. 3A). Control P14 male mice were unilaterally injected with AAV-tdTomato $\left(1.16 \times 10^{10} \mathrm{VPs}\right)$, and experimental animals with AAV-ephrin-B1 $\left(3.78 \times 10^{10} \mathrm{VPs}\right)$. At P28, mice were subjected to immunohistochemistry and whole-cell electrophysiology experiments. There was a significant increase in ephrin-B1 immunoreactivity in all three layers of CA1 hippocampus on the AAV-ephrinB1 injected side (ipsilateral side, OE) compared with control noninjected (contralateral side, Fig. 3B-D; Extended Data Fig. 3-1; SO: $t$ test, $t_{(21)}=5.366, p<0.0001$; SP: $t$ test, $t_{(21)}=6.220, p<0.0001$; SR: $t$ test, $\left.t_{(21)}=4.062, p=0.0006\right)$.

For whole-cell voltage-clamp electrophysiology, I/O curves were generated and stimulation that induced the maximum response was used to measure AMPAR- and NMDAR-mediated EPSCs. We noted higher amplitude of evoked AMPAR- and NMDAR-mediated responses in control slices from AAVtdTomato-injected mice (control group for OE mice; Fig. 3E,F) compared with control slices from the ERT2-Cre ${ }^{G F A P}$ mouse line (control group for $\mathrm{KO}$ mice; Fig. $2 B, C$ ), potentially because of the effects of viral injection or the differences between mouse lines. Therefore, comparisons were made only between the $\mathrm{AAV}$-injected $\mathrm{OE}$ group and the corresponding control group. Recordings from the AAV-ephrin-B1-injected ipsilateral side were included in the OE group (Fig. $3 A$ ). No significant differences were observed between recordings from the contralateral

indicating potentially no effect on presynaptic activity (Extended Data Fig. 3-2). $\boldsymbol{K}$, The cumulative distribution of mEPSC amplitude shows a significant leftward shift (smaller mESPC amplitude across the distribution) for $\mathrm{OE}$ (dotted dark gray) compared with control group (gray). L, Average amplitude of mEPSCs was lower in $\mathrm{OE}$ compared with control group (Extended Data Fig. 3-2). Graphs represent mean. Error bars indicate SEM. $t$ test: ${ }^{*} p<0.05$; ${ }^{* *} p<0.01$ noninjected side of AAV-ephrin-B1-injected mice and the ipsilateral side of AAV-tdTomato-injected mice; both were combined in the control group (Fig. $3 A$ ). A significant reduction of evoked AMPAR- and NMDAR-mediated responses was observed in the OE group compared with control (Fig. 3E,F; Extended Data Fig. 3-2; AMPAR: $t$ test, $t_{(23)}=2.692, p=0.0130$; NMDAR: $t$ test, $\left.t_{(20)}=3.573, p=0.0019\right)$, with no effect to AMPAR/NMDAR EPSC ratio (Fig. 3G; Extended Data Fig. 3-2; $t$ test, $\left.t_{(20)}=0.9733, p=0.3426\right)$. However, mEPSCs were unchanged in the OE group compared with control (Fig. $3 H$ ), including cumulative probability of interevent interval (Fig. 3H,I; K-S test, $n=550$ and 360 for control and OE group, respectively, $p=0.4819, \mathrm{D}=0.1408$ ) and average frequency of mEPSCs (Fig. 3 J; Extended Data Fig. 3-2; $t$ test, $\left.t_{(12)}=1.036, p=0.3206\right)$. In contrast, the OE group exhibited a significant leftward shift in cumulative probability of mEPSC amplitude, showing a reduced number of larger events with $>10 \mathrm{pA}$ amplitude (Fig. $3 \mathrm{~K}$; K-S test, $n=550$ and 360 for control and OE group, respectively, $p=0.0145, \mathrm{D}=0.1065)$ and a reduced average mEPSC amplitude compared with their control counterparts (Fig. 3L; Extended Data Fig. 3-2; $t$ test, $\left.t_{(12)}=1.821, p=0.0468\right)$. In the OE group, the reduced number of the events with large amplitude may potentially indicate a reduced strength or loss of synapses at the soma or proximal dendrites of CA1 hippocampal neurons, in a close proximity to recording electrode.

Together, this further confirms that astrocytic ephrin-B1 negatively affects excitatory synaptic transmission in the developing hippocampus, such that the loss of ephrin-B1 in astrocytes enhances, but its OE reduces excitatory responses in CA1 hippocampal neurons.

\section{Excessive excitatory synapse formation is observed in CA1 hippocampus of developing $\mathrm{KO}$ mice}

Next, we examined whether loss of ephrin-B1 from astrocytes would also affect the number of excitatory synapses in CA1 hippocampus by coimmunostaining against presynaptic vGlut 1 and postsynaptic PSD95 using brain slices from Group $1 \mathrm{KO}$ and their control mice (Fig. $4 A, B$ ). We observed a significant increase in vGlut1-positive puncta (Fig. $4 C$; Extended Data Fig. 4-1; $t$ test, $t_{(21)}=4.238, p=0.0004$ ), PSD95-positive puncta (Fig. 4D; Extended Data Fig. $4-1$; $t$ test, $\left.t_{(17)}=2.801, p=0.0123\right)$, and vGlut1/PSD95 colocalization (Fig. $4 E$; Extended Data Fig. $4-1 ; t$ test, $\left.t_{(51)}=3.784, p=0.0004\right)$ in the SR but not SLM layer of CA1 hippocampus of KO mice compared with control mice. Further, dendritic spine density was significantly higher in $\mathrm{KO}$ compared with control (Fig. 4G; Extended Data Fig. 4-2; $t$ test, $t_{(31)}=2.78$, $p=0.0092$ ). Together, our results suggest that the loss of astrocytic ephrin-B1 results in excessive excitatory synapse formation on excitatory CA1 neurons, which may contribute to enhanced excitability.

\section{Developmental astrocyte-specific deletion of ephrin-B1 affected size of dendritic spines but not synaptic levels of AMPARs}

To assess synapse maturation, spine morphology was assessed using Group 3 mice. Dendritic spines were identified with GFP in Thy1-GFP-ERT2-Cre ${ }^{\text {GFAP }}$ ephrin-B1 ${ }^{\text {flox/y }}$ (KO) or Thy1GFP-ERT2-Cre ${ }^{G F A P}$ (Control) male mice expressing GFP in hippocampal pyramidal neurons (Fig. $4 F$ ). Spine length was comparable between control and $\mathrm{KO}$ mice (Fig. $4 H$; Extended Data Fig. 4-2; $t$ test, $\left.t_{(31)}=0.0697, p=0.9449\right)$. Interestingly, $\mathrm{KO}$ mice had a greater proportion of spines with smaller heads 

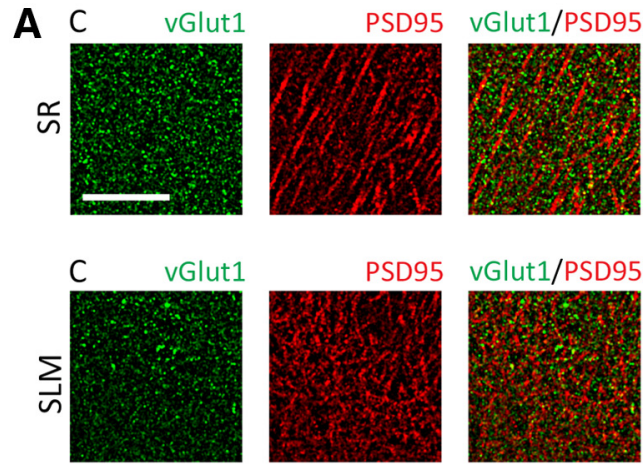

C vGlut1 immunoreactivity

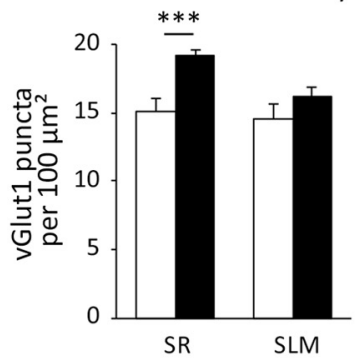

D PSD95 immunoreactivity

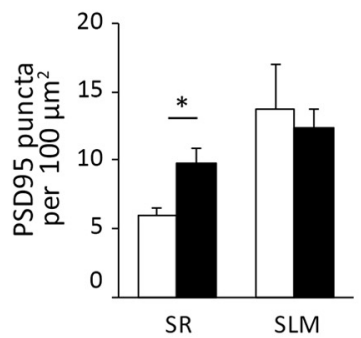

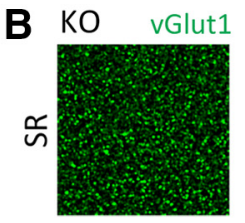
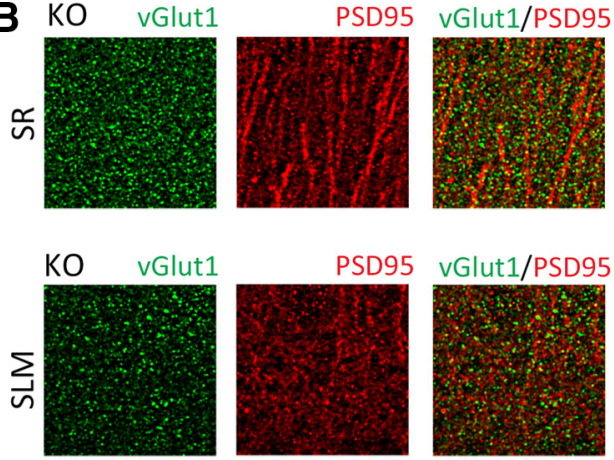

E vGlut1/PSD95 colocalization

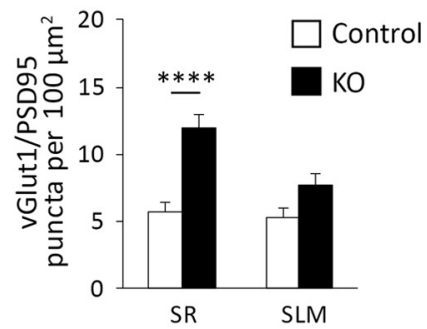

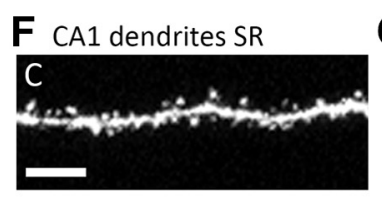

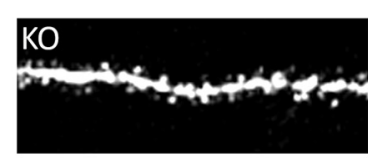

G Spine Density
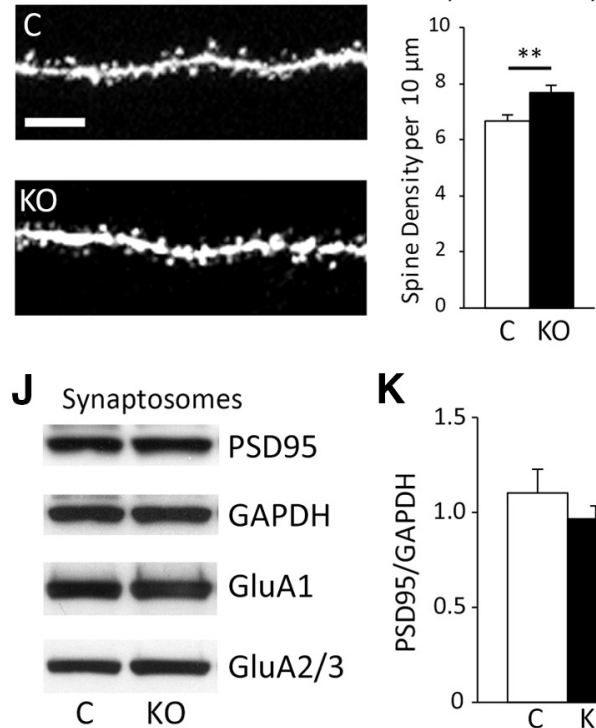

K

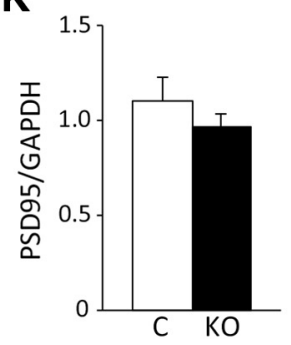

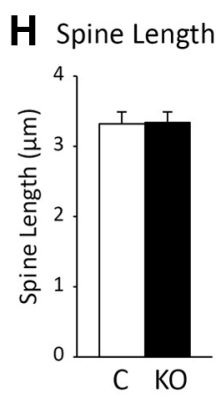

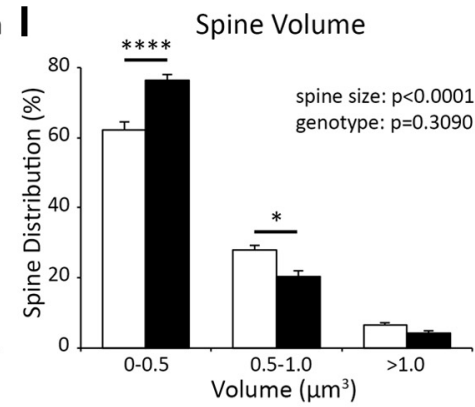

Figure 4. Early postnatal astrocyte-specific deletion of ephrin-B1 resulted in increased number of excitatory synapses in CA1 hippocampus. $A, B$, Confocal images represent vGlut1 (green) and PSD-95 (red) immunolabeling in SR and SLM areas of CA1 hippocampus of Group 1 control and K0 mice. Scale bar, $25 \mu \mathrm{m}$. C-E, Graphs represent density of vGlut1-positive puncta (C), PSD95-positive puncta (D), and vGlut1/PSD95 colocalization (E) per $100 \mu \mathrm{m}^{2}$ of SR and SLM areas in CA1 hippocampus of control and K0 mice $(n=3$ or 4 mice; Extended Data Fig. 4-1). K0 mice showed a significant increase of vGlut1-positive puncta, PSD95-positive puncta, and the colocalization of vGlut1 and PSD95 in the SR CA1 hippocampus. Graphs represent mean. Error bars indicate SEM. ${ }^{* *} p<0.001$. ${ }^{* * *} p<0.0001$. $\boldsymbol{F}$, Confocal images represent dendrites of CA1 neurons expressing GFP in the SR area of CA1 hippocampus of control and K0 mice. Scale bar, $10 \mu \mathrm{m}$. $\mathbf{G}-\mathbf{I}$, Graphs represent average density of dendritic spines per $10 \mu \mathrm{m}$ dendrite $(\boldsymbol{G})$, average spine length $(\boldsymbol{H})$, and spine volume $(\boldsymbol{I})$. There was a significant increase in average dendritic spine density in $\mathrm{KO}$ mice compared with control mice (Extended Data Fig. 4-2). There was a significantly increased proportion of dendritic spines with smaller heads (volume $0-0.5 \mu \mathrm{m}^{3}$ ), a decreased percent of medium-size spines (volume $0.5-1.0 \mu \mathrm{m}^{3}$ ), and same percent of large, mature spines (volume $>1.0 \mu \mathrm{m}^{3}$ ) observed in $\mathrm{KO}$ mice compared with control mice. Graphs represent mean. Error bars indicate SEM. ${ }^{*} p<0.05 ;{ }^{* *} p<0.01 ;{ }^{* * *} p<0.0001$ (Extended Data Fig. 4-3). J, Western blots show levels of PSD-95, GAPDH, and AMPAR subunits (GluA1 and GluA2/3) in synaptosomes isolated from hippocampus of P28 control and K0 mice (Extended Data Fig. 4-4). $\boldsymbol{K}$, Graphs represent mean ratios of synaptic PSD-95 to GAPDH, and GluA1, or GluA2/3 levels to PSD-95 levels in synaptosomes isolated from P28 hippocampus of control and K0 mice (Extended Data Fig. 4-5). AMPAR levels at hippocampal synapses are similar in P28 control and KO mice. Graphs represent mean. Error bars indicate SEM.

(0-0.5 $\mu^{3}$; Extended Data Fig. 4-3; two-way ANOVA, Tukey's post hoc test, $p<0.0001$ ) and a smaller percent of medium size spines $\left(0.5-1.0 \mu \mathrm{m}^{3}\right.$; Fig. $4 I$; Extended Data Fig. 4-3; two-way ANOVA, Tukey's post hoc test, $p=0.0162)$ but similar levels of large, more mature spines $\left(>1.0 \mu \mathrm{m}^{3}\right.$; Fig. $4 \mathrm{I}$;
Extended Data Fig. 4-3; two-way ANOVA, Tukey's post hoc test, $p=0.9617$ ) compared with control animals.

In addition, the levels of synaptic AMPAR subunits GluA1 and GluA2/3 were analyzed in developing hippocampus of control and $\mathrm{KO}$ mice. Crude synaptosomes were isolated from P28 
A Whole Cell Recordings

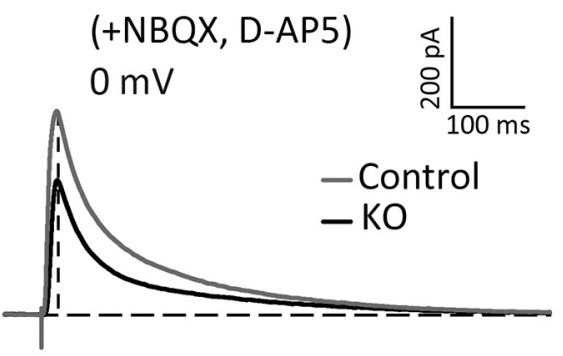

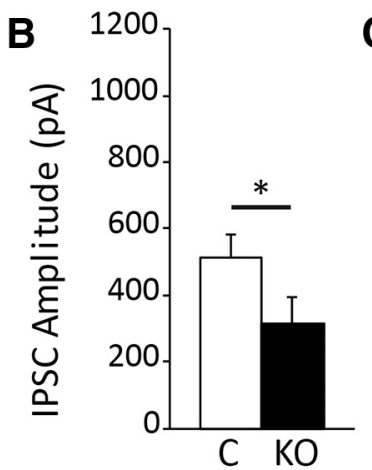

C $\operatorname{mIPSC}(+\mathrm{NBQX}, \mathrm{D}-\mathrm{AP} 5, \mathrm{TTX})$

Control

咅

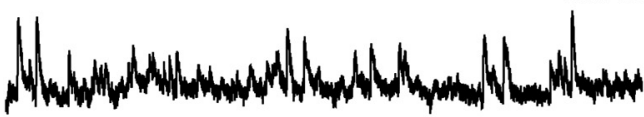

$\mathrm{KO}$

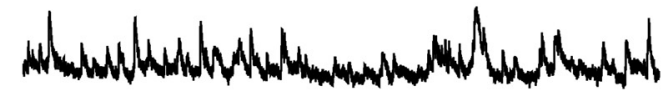

D

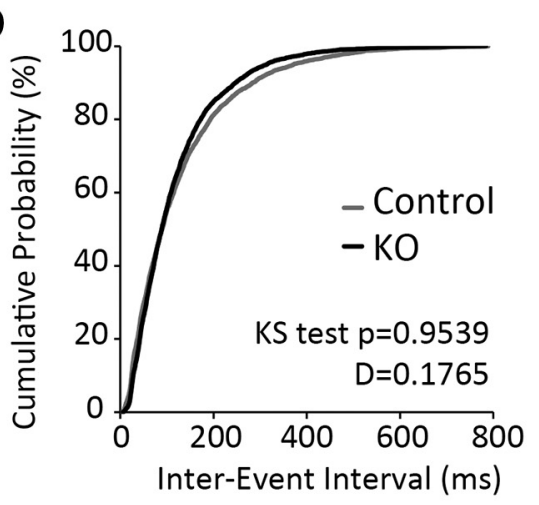

H

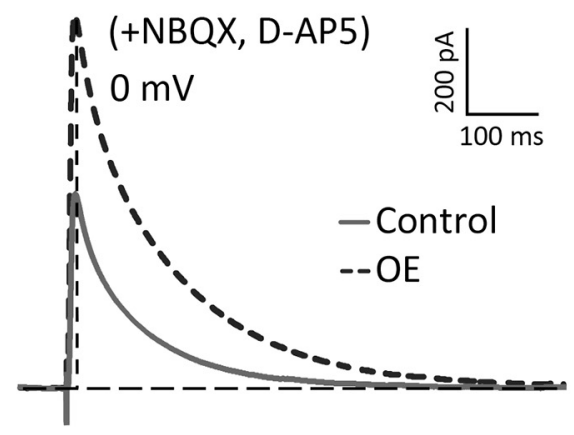

E

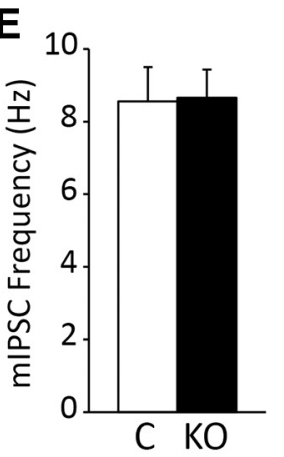

F

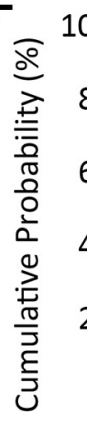

G

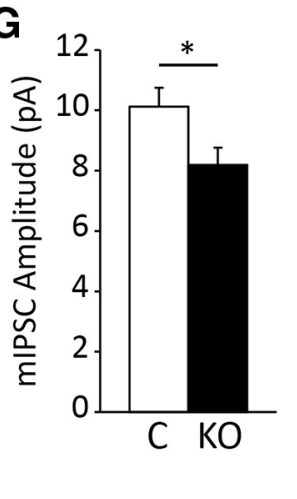

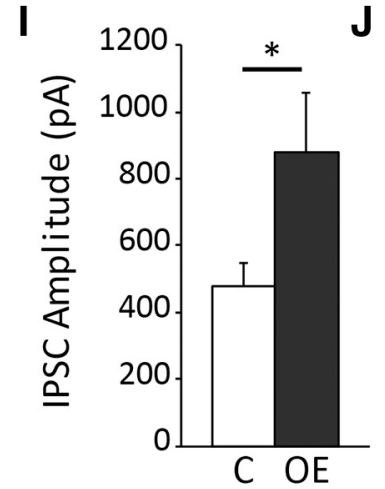

$\mathbf{J} \operatorname{mIPSC}(+\mathrm{NBQX}, \mathrm{D}-\mathrm{AP5}, \mathrm{TTX})$

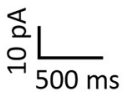

Control

- Control

$-\mathrm{KO}$

KS test $p<0.0001$ $D=0.0541$

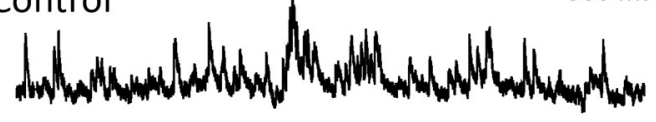

OE

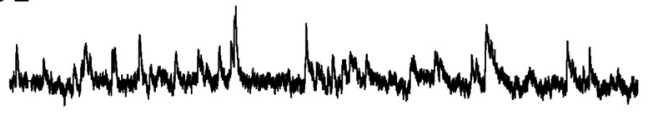

$\mathbf{K}$

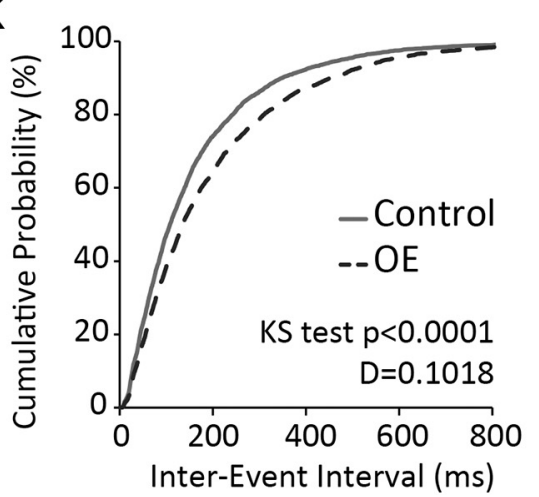

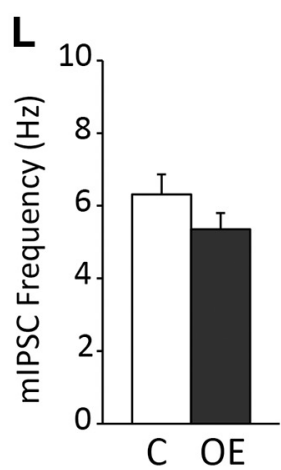

M

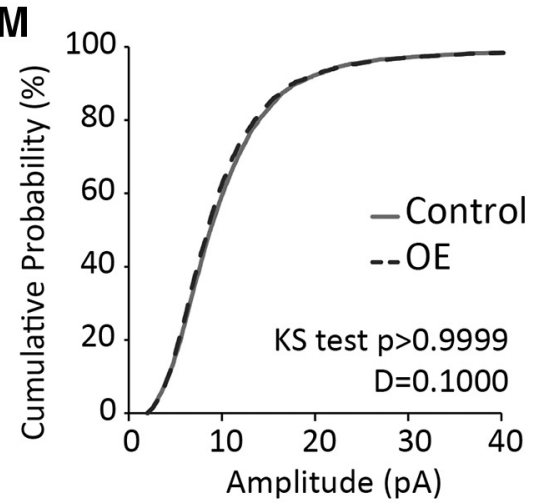

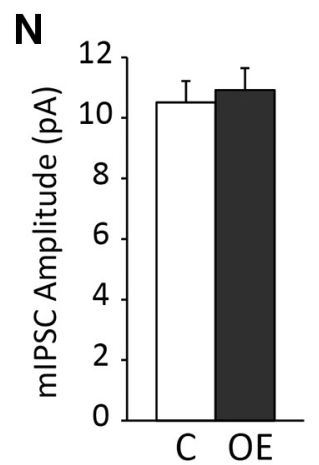

Figure 5. Inhibition is altered in CA1 hippocampal neurons following deletion or OE of astrocytic ephrin-B1 during early postnatal development. $A$, Representative traces showing evoked IPSCs recorded in CA1 pyramidal neurons from Group 1 control (gray) and K0 (black); whole-cell recording was done in the presence of D-AP5 and NBQX to block AMPAR- and NMDAR-mediated currents. B, Graph represents average amplitude of evoked IPSCS ( $n=12-14$ cells, 6 mice per group; Extended Data Fig. 5-1). Amplitude of evoked IPSCs is significantly decreased in CA1 neurons of P28 KO mice compared with control mice. $C$, Representative traces of mIPSCs in control and KO mice; whole-cell recording was done in the presence of NBQX, D-AP5, and TTX ( $n=12-14$ cells, 6 mice per group). $\boldsymbol{D}$, The cumulative distribution of mIPSC interevent intervals in control (gray) and K0 (black) mice. $\boldsymbol{E}$, Average frequency of mIPSCs was not significantly different between control and KO mice, indicating potentially no effect on presynaptic activity (Extended Data Fig. 5-1). $\boldsymbol{F}$, The cumulative distribution of mIPSC amplitude shows a significant leftward shift (smaller mIPSC amplitude across the distribution) for KO (black) mice compared with control (gray). G, Average amplitude of mIPSCs was significantly lower in KO compared with control mice (Extended Data Fig. 5-1). H, Representative traces showing evoked IPSCs recorded in CA1 pyramidal neurons from P28 control (gray) and 0E (dotted dark gray); whole-cell recording was done in the presence of D-AP5 and NBQX to block AMPAR- and NMDAR-mediated currents. I, Graph represents average amplitude of evoked IPSCs ( $n=10$ or 11 cells, 6 mice per group; 
hippocampi of control and KO mice (Group 1) and analyzed with immunoblotting (Fig. 4J). Significant enrichment of PSD95 and synapsin 1 in synaptosome fraction compared with lysates was also confirmed by immunoblotting (see Extended Data Fig. 4-4). Synaptic PSD95 levels (Fig. 4K; Extended Data Fig. 4-5, $t$ test, $\left.t_{(10)}=0.9338, p=0.3724\right)$ and levels of AMPAR subunits GluA1 (Fig. $4 K$; Extended Data Fig. $4-5, t$ test, $t_{(10)}=1.085$, $p=0.3036$ ) and GluA2/3 (Fig. $4 K$; Extended Data Fig. 4-5, $t$ test, $t_{(10)}=0.1792, p=0.8613$ ) were not significantly different between control and KO mice. These results are consistent with similar AMPAR/NMDAR EPSC ratio that was observed in control and $\mathrm{KO}$ mice (Fig. 2D), suggesting no differences in the functional maturation of excitatory synapses between control and $\mathrm{KO}$ mice.

Despite no effect on functional maturation of excitatory synapses, KO mice exhibited a larger proportion of dendritic spines with smaller heads, suggesting changes in structural maturation.

Inhibitory postsynaptic currents are reduced in CA1 neurons of astrocytic ephrin-B1 KO mice, while OE enhances evoked IPSCs in CA1 hippocampus

To determine whether astrocytic ephrin-B1 affects inhibitory synapses, IPSCs were recorded from CA1 hippocampal neurons in brain slices from Group $1 \mathrm{KO}$ and their control mice using whole-cell voltage-clamp electrophysiology (Fig. 5A). In the presence of NMDAR and AMPAR blockers, D-AP5 and NBQX, we observed a significant decrease in evoked IPSC amplitude in CA1 hippocampal neurons of $\mathrm{KO}$ mice compared with control (Fig. 5B; Extended Data Fig. $5-1$; $t$ test, $\left.t_{(20)}=1.90, p=0.0360\right)$. However, the cumulative probability of interevent interval (Fig. $5 D$; K-S test, $n=3500$ and 4500 for control and KO group, respectively, $p=0.1765, \mathrm{D}=0.9539$ ) and average frequency of mIPSCs was unchanged in KO group compared with control (Fig. 5E; Extended Data Fig. 5-1; $t$ test, $t_{(13)}=0.02485$, $p=0.9806)$. KO mice exhibited a significant leftward shift of cumulative probability of mIPSC amplitude (Fig. 5F; K-S test, $n=3500$ and 4500 for control and KO group, respectively, $p<0.0001, \mathrm{D}=0.0541)$ and a reduced average mEPSC amplitude compared with their control counterparts (Fig. 5G; Extended Data Fig. $5-1$; $t$ test, $\left.t_{(13)}=0.7904, p=0.04435\right)$. Reduced proportion of high-amplitude $(>10 \mathrm{pA})$ events in the KO group may suggest a reduced strength or loss of inhibitory synapses on the cell body or proximal dendrites of CA1 hippocampal neurons (in a close proximity to the recording electrode).

Conversely, to determine whether the OE of astrocytic ephrin-B1 affects inhibitory synapses, IPSCs were recorded from CA1 hippocampal neurons on AAV-ephrin-B1-injected ipsilateral side (OE; Fig. $3 A$ ) using whole-cell voltage-clamp electrophysiology (Fig. $5 H-N$ ). Control group included recordings from both contralateral noninjected side of AAV-ephrin-B1injected mice and ipsilateral side of AAV-tdTomato-injected

\footnotetext{
Extended Data Fig. 5-2). Amplitude of evoked IPSCs was significantly decreased in CA1 neurons of OE compared with control group. J, Representative traces of mIPSCs in P28 control and OE groups; whole-cell recording was done in the presence of NBQX, D-AP5, and TTX ( $n=10$ or 11 cells, 6 mice per group). $\boldsymbol{K}$, The cumulative distribution of mIPSC interevent intervals in control (gray) and $\mathrm{OE}$ (dotted dark gray) groups. There is a significant rightward shift in $\mathrm{OE}$ group, indicating larger interevent intervals between spikes. $L$, However, average frequency of mIPSCs was not significantly different between control and $0 \mathrm{E}$ groups (Extended Data Fig. 5-2). M, The cumulative distribution of mIPSC amplitude in control (gray) compared with $\mathrm{OE}$ group (dotted dark gray). $\boldsymbol{N}$, Average amplitude of mIPSCs was similar between control and OE groups (Extended Data Fig. 5-2). Graphs represent mean. Error bars indicate SEM. $t$ test: ${ }^{*} p<0.05$.
}

$\leftarrow$ mice (Fig. $3 A$ ), as there were no significant differences observed between these groups. Interestingly, we observed a significant increase in evoked IPSC amplitude in CA1 hippocampal neurons of the OE group compared with control (Fig. 5I; Extended Data Fig. $5-2$; $t$ test, $\left.t_{(19)} ;=2.135 p=0.0230\right)$. The cumulative probability of interevent interval exhibited a rightward shift in $\mathrm{OE}$ mice (Fig. $5 K$; K-S test, $n=4800$ and 4100 for $\mathrm{C}$ and OE group, respectively, $p<0.0001, \mathrm{D}=0.1018$ ); however, the average frequency of mIPSCs was unchanged in the OE group compared with control (Fig. $5 L$; Extended Data Fig. $5-2 ; t$ test, $t_{(24)}=1.32, p=0.1988$ ). The cumulative probability of mIPSC amplitude (Fig. 5M; K-S test, $n=4800$ and 4100 for control and OE group, respectively, $p>0.9999, \mathrm{D}=1.000)$, and average mEPSC amplitude was similar between the OE and control groups (Fig. $5 N$; Extended Data Fig. 5 - 2 ; $t$ test, $\left.t_{(24)}=0.39, p=0.6999\right)$.

Our results show that loss of ephrin-B1 from astrocytes during P14-P28 developmental period leads to reduced evoked IPSCs and mIPSC amplitude in CA1 hippocampal neurons, but OE of ephrin-B1 in astrocytes resulted in enhanced evoked IPSCs without affecting mIPSC amplitude

\section{Changes in the density of PV-positive inhibitory neurons in CA1 hippocampus may contribute to impaired inhibition in KO mice}

To determine whether astrocyte-specific deletion of ephrin-B1 affects the inhibitory drive onto CA1 hippocampal neurons, inhibitory synaptic sites were detected on GFP-expressing dendrites of CA1 excitatory neurons with immunostaining against VGAT and gephyrin in brain slices from Group $3 \mathrm{KO}$ male mice and their controls (Fig. 6A,B). We observed no effect of ephrinB1 deletion from developing astrocytes on VGAT/gephyrin colocalized puncta along the first-order dendrites (Fig. 6C; Extended Data Fig. 6-1; $t$ test, $t_{(51)}=1.449, p=0.1534$ ) or second-order dendrites (Fig. 6C; Extended Data Fig. 6-1; $t$ test, $t_{(22)}=0.7795$, $p=0.4440$ ) of CA1 neurons in SR area of the hippocampus. However, we did observe a significant decrease in VGAT/ gephyrin colocalization on the cell bodies of CA1 neurons in SP area (Fig. 6D; Extended Data Fig. 6-1; $t$ test; $t_{(60)}=2.030$, $p=0.0468)$ and the dendrites of the CA1 neurons in SO area (Fig. $6 E$; Extended Data Fig. $6-1$; $t$ test, $t_{(18)}=2.307, p=0.0332$ ).

We also observed a significant twofold decrease in the density of PV-positive inhibitory neurons in the CA1 hippocampus of $\mathrm{KO}$ mice compared with control mice in SO (Fig. 6G; Extended Data Fig. 6-2; $t_{(66)}=2.889, p=0.0052$ ), SP (Fig. 6G; Extended Data Fig. 6-2; $t$ test, $t_{(66)}=4.595, p<0.0001$ ), and SR (Fig. 6G; Extended Data Fig. 6-2; $t$ test, $t_{(66)}=4.727, p<0.0001$ ) layers of CA1 hippocampus. Interestingly, vGlut1-positive excitatory presynaptic boutons onto PV inhibitory neurons were also reduced in KO mice, specifically in the SO (Fig. 6I; Extended Data Fig. 62; $t$ test, $t_{(48)}=5.536, p<0.0001$ ) and SP (Fig. 6I; Extended Data Fig. 6-2; $t$ test, $t_{(67)}=6.349, p<0.0001$ ), but not in the SR (Fig. 6I; Extended Data Fig. 6-2; $t$ test, $t_{(20)}=0.2142, p=0.8325$ ) layers of CA1 hippocampus. The reduced excitatory drive onto PV inhibitory neurons in the CA1 hippocampus may contribute to the reduced number of PV-expressing cells, lower number of inhibitory synapses in the SP and SO layers of the CA1 hippocampus, and lower inhibitory activity, resulting in an overall increase in $\mathrm{E} / \mathrm{I}$ balance in astrocyte-specific ephrin-B1 KO mice.

\section{Astrocytic ephrin-B1 KO mice show impaired social behaviors, but no anxiety or hyperactivity}

Altered E/I balance as a result of aberrant E/I synapse development is also observed in several ASD mouse models (Lee et al., 
A Control GFP VGAT Geph

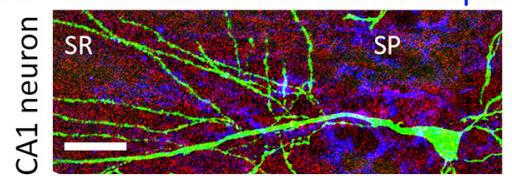

CA1 dendrite SR
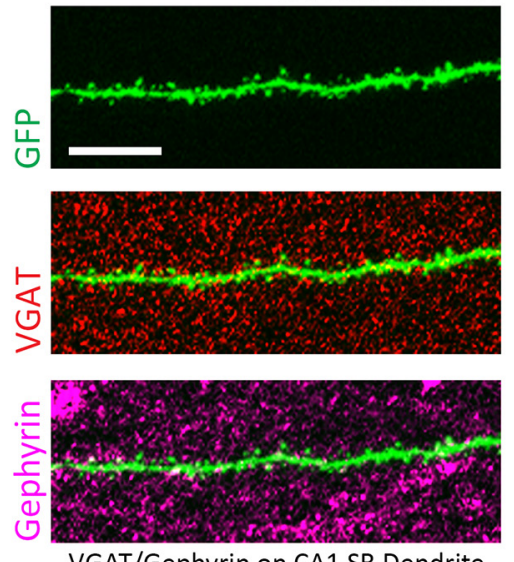

VGAT/Gephyrin on CA1 SR Dendrite
First Order Dendrite Second Order Dendrite

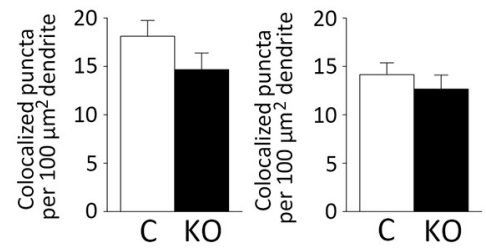

F Control

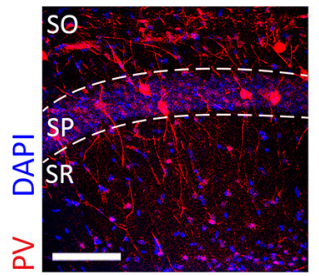

$\mathrm{KO}$
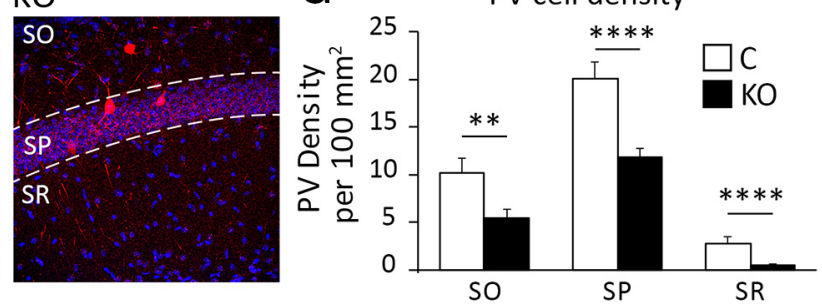

H C

SP

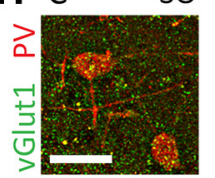

$\mathrm{KO}$

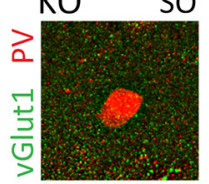

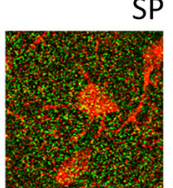

SP

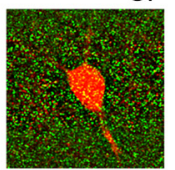

G
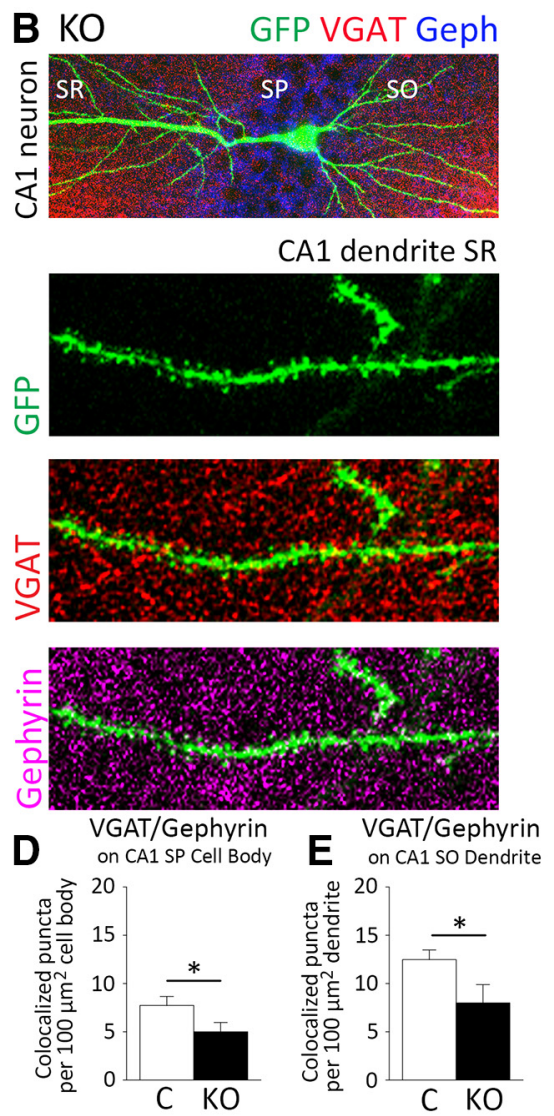

E

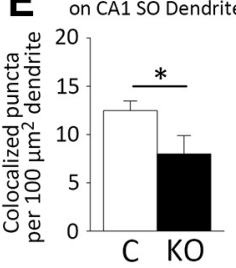

PV cell density

vGlut1/PV colocalization

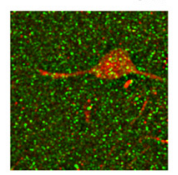

SR I

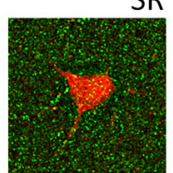

$\mathrm{SR}$
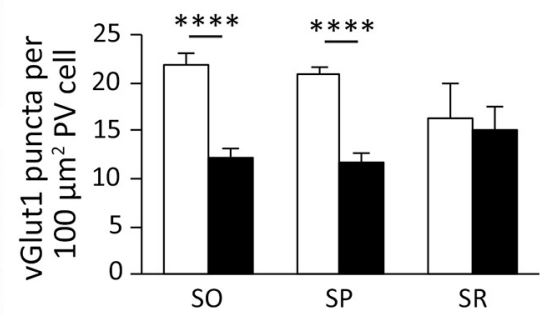

Figure 6. Inhibitory synaptic sites on the CA1 pyramidal neurons and the density of PV-expressing inhibitory neurons are reduced following the deletion of astrocytic ephrin-B1 during early postnatal development. $\boldsymbol{A}, \boldsymbol{B}$, Confocal images represent CA1 excitatory neurons expressing GFP (green) costained with VGAT (red) and gephyrin (blue) to visualize inhibitory synaptic sites. Scale bar, $50 \mu \mathrm{m}$. High-magnification images represent GFP-expressing dendrites (green) with VGAT (red) and gephyrin (purple). Scale bar, $10 \mu \mathrm{m}$. $\mathbf{C}-\boldsymbol{E}$, Graphs represent VGAT/gephyrin colocalized puncta on GFP-expressing primary and secondary SR dendrites $(\boldsymbol{C})$, neuronal cell bodies in SP $(\boldsymbol{D})$ and S0 dendrites $(\boldsymbol{E})$ of CA1 neurons. K0 mice exhibited a significant decrease in density of VGAT/gephyrin colocalized puncta on CA1 neurons in the SP and S0 layers ( $n=11-20$ images; 4 mice per group, $t$ test $p<0.05$; Extended Data Fig. 6-1). $\boldsymbol{F}$, Confocal images represent PV-expressing cells (red) and DAPI staining (blue) to identify CA1 hippocampal layers, S0, SP, and SR. G, Graph represents density of PV-expressing neurons in S0, SP, and SR layers of CA1 hippocampus. KO mice exhibited a significant decrease in density of PV-expressing inhibitory neurons in all three layers $(n=30$ 35 images; 3 mice per group; $t$ test $p<0.01, p<0.0001$; Extended Data Fig. 6-2). $\boldsymbol{H}$, Confocal images represent vGlut1 immunoreactivity (green) on PV-expressing cells (red) in the SO, SP, and SR layers of the CA1 hippocampus. Scale bar, $100 \mu \mathrm{m}$. I, Graphs represent colocalization of vGlut1 puncta and PV immunoreactivity in the SO, SP, and SR layers of the CA1 hippocampus ( $n=10-40$ cells, 3 mice per group; $t$ test $p<0.0001$; Extended Data Fig. 6-2). K0 mice exhibit decreased numbers of excitatory
2017) and may underlie changes in ASD-like behaviors, such as social novelty and preference, as well as anxiety and hyperactivity. As deletion of astrocytic ephrin-B1 occurs globally in $\mathrm{KO}$ mice generated in this study, it is possible that the changes in $\mathrm{E} / \mathrm{I}$ balance that we see in the hippocampus may not be exclusive to this area of the brain. Considering that ephrins and Eph receptors are identified as risk genes for the development of ASD in humans (Sanders et al., 2012), we also analyzed ASD-like behaviors. Moreover, social memory has been linked to hippocampus function as well (Hitti and Siegelbaum, 2014; Ko, 2017). Social novelty and social preference were assessed using a three-chamber test using Group $1 \mathrm{KO}$ male mice and their controls. Mice were placed in a cage containing two side chambers and were tested in two $10 \mathrm{~min}$ sessions. In Session 1, an unfamiliar stranger mouse (S1) was placed in one of the side chambers, with the other chamber remaining empty (Fig. 7A). Control mice spent significantly more time in the chamber with S1 than the empty chamber (two-way ANOVA, Tukey's post hoc test, $p<0.0001$ ) or the middle chamber (two-way ANOVA, Tukey's post hoc test, $p=0.0002$ ); however, KO mice spent a similar amount of time in each chamber, indicating impaired social preference (Fig. 7B; Extended Data Fig. 7-1). In Session 2, a novel mouse (S2) was placed in the empty chamber, while now familiar S1 mouse was remained in the same chamber. This test assessed social preferences by measuring the time that the mouse spent with either the familiar S1 mouse or a novel S2 mouse (Fig. 7A). Control mice spent significantly more time in the chamber with the novel S2 mouse than the familiar S1 mouse (two-way ANOVA, Tukey's post hoc test, $p=$ 0.0005 ) or the middle chamber (twoway ANOVA, Tukey's post hoc test, $p=0.0080$, Fig. $7 C$; Extended Data Fig. 7-2). However, KO mice spent the same amount of time in the chamber containing S1 mouse, S2 mouse, or the middle chamber, suggesting they could not discriminate between a familiar and novel

\footnotetext{
vGlut1-positive boutons on PV-expressing inhibitory neurons in the SO and SP of CA1 hippocampus. Graphs represent mean. Error bars indicate SEM. ${ }^{*} p<0.05$, ${ }^{* *} p<0.01$, ${ }^{* * * *} p<0.0001$.
} 
A Three Chamber Test

Social Preference

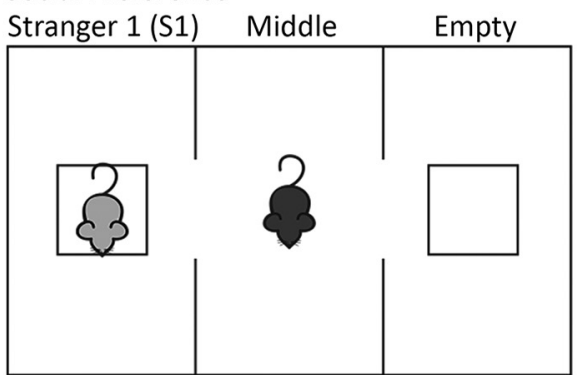

B

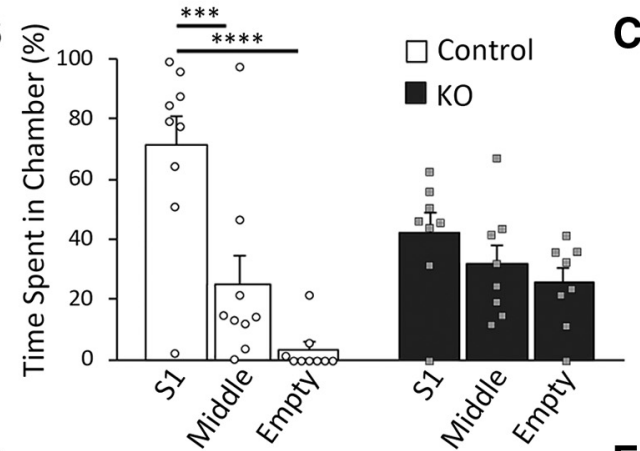

D

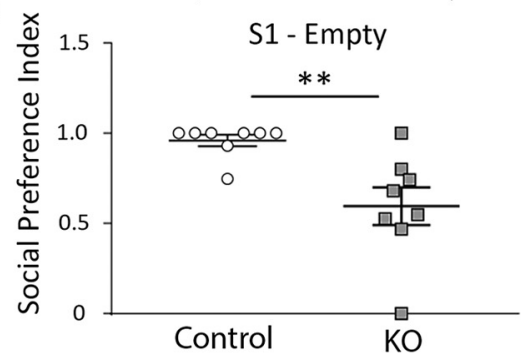

$\mathbf{F}$

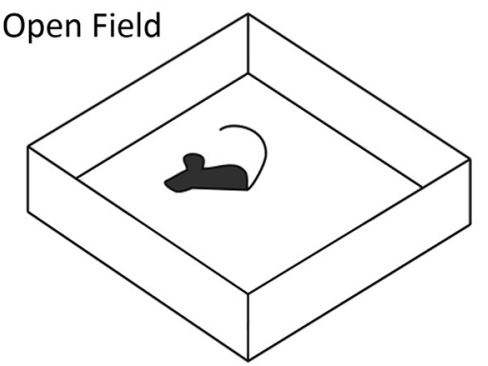

E

G
Social Novelty

Stranger 1 (S1) Middle Stranger 2(S2)

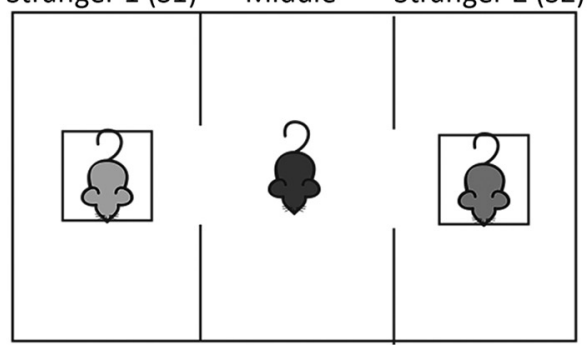

C
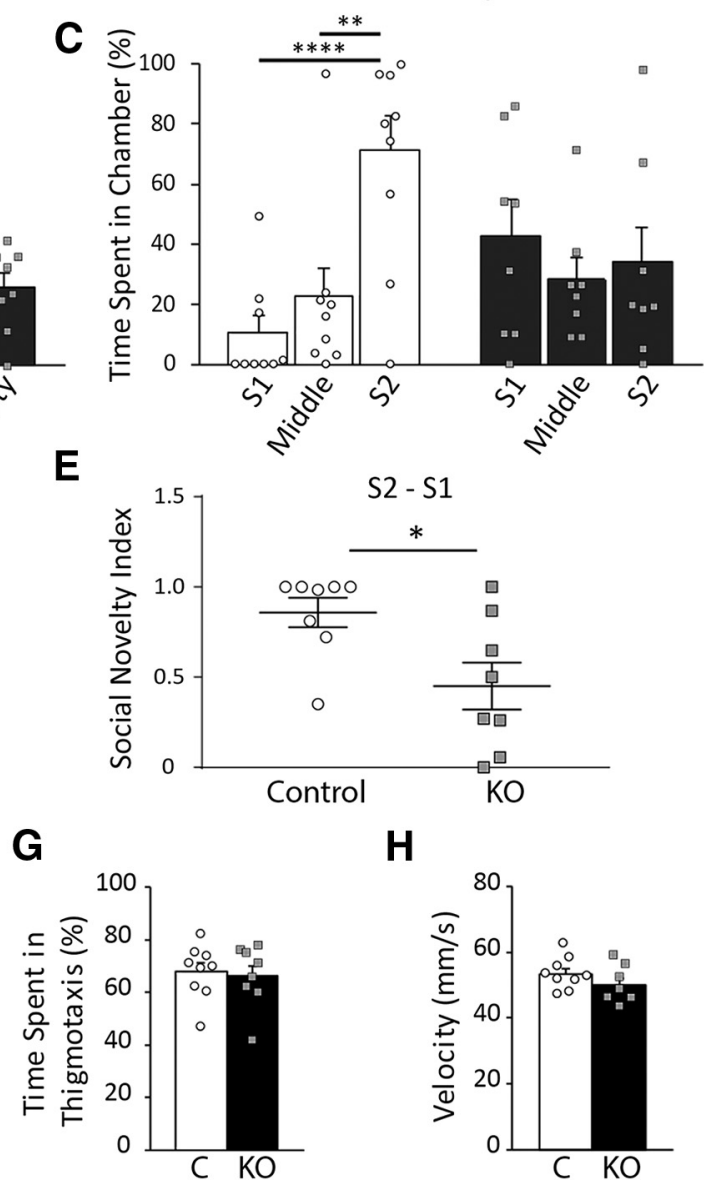

H

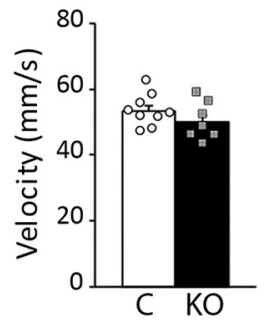

Figure 7. Ablation of astrocytic ephrin-B1 during early postnatal development affected mouse social behaviors. $A$, Diagram of three-chamber test for social preference and social novelty. Mice were placed in the middle chamber containing two side chambers and were tested in two 10 min sessions. During social preference test, a stranger mouse (S1) was placed in one of the side chambers, with the other chamber remaining empty. During social novelty test, now familiar stranger mouse (S1) remained in the side chamber, while a novel mouse (S2) was placed in the empty chamber. $\boldsymbol{B}$, Graph represents time spent in either three chambers during social preference test. Control mice prefer spending time with S1 mouse compared with time in the middle (two-way ANOVA, Tukey's post hoc test, $p=0.0002$ ) or empty chamber (two-way ANOVA, Tukey's post hoc test, $p<0.0001$; Extended Data Fig. 7-1). K0 mice show impaired sociability and spend the same amount of time in each chamber. C, Graph represents time spent in each chamber during social novelty test. Control mice spend significantly more time with novel 52 mouse than with familiar S1 mouse (two-way ANOVA, Tukey's post hoc test, $p=0.0005$ ), or in the middle chamber (two-way ANOVA, Tukey's post hoc test, $p=0.0080$; Extended Data Fig. 7-2), indicating normal social novelty. KO mice spend the same amount of time in S1, middle, or S2 chambers. D, Graph represents social preference index, calculated as $\left(\frac{\text { time in } \mathrm{S1} \text { chamber }}{\text { time in } \mathrm{S1} \text { chamber }+ \text { time in empty chamber }}\right)$. K0 mice show a significant reduction in social preference index $(t$ test, $p=0.0049)$, with a value near 0.5 indicating time spent between S1 chamber and the empty chamber was nearly equal (Extended Data Fig. 7-3). E, Graph represents social novelty index, calculated as $\left(\frac{\text { time in S2 chamber }}{\text { time in S2 chamber }+ \text { time in S1 chamber }}\right)$. K0 mice show a significant reduction in social novelty index ( $t$ test, $p=0.0186$ ), with a value near 0.5 indicating equal time spent between $S 2$ and $S 1$ chambers (Extended Data Fig. 7-3). $\boldsymbol{F}$, Schematics of open field test; during testing animals were allowed to freely explore the open field for 10 min while time spent in thigmotaxis, and average velocity were measured. $\boldsymbol{G}$, Graph represents percent time spent in thigmotaxis with no significant differences between control and K0 mice ( $t$ test, $t_{(15)}=0.3455, p=0.7345$; Extended Data Fig. 7-4). $\boldsymbol{H}$, Graph represents average velocity of control and $\mathrm{KO}$ mice $\left(t\right.$ test, $t_{(15)}=0.1 .214, p=0.2435$; Extended Data Fig. 7-4). Graphs represent mean. Error bars indicate SEM. ${ }^{*} p<0.05,{ }^{* *} p<0.01$, ${ }^{* * *} p<0.001,{ }^{* * * 0} p<0.0001$. 
mouse. Further, the social preference and social novelty index was calculated to directly measure the differences between control and KO mice; KO mice had a significantly lower social preference index (Fig. $7 D$; Extended Data Fig. $7-3$; $t$ test, $t_{(14)}=3.337, p=0.0049$ ). A value near 1 indicates more time spent with the $S 1$ chamber, while a value near 0.5 indicates equal amount of time spent in both $\mathrm{S} 1$ and empty chamber. The social preference index of $\mathrm{KO}$ was near 0.5 , indicating impaired social preference, spending equal amount of time between an empty chamber and a chamber with a stranger mouse. The social novelty index was also significantly lower in $\mathrm{KO}$ mice (Fig. 7E; Extended Data Fig. 7-3; $t$ test, $t_{(14)}=2.661, p=$ $0.0186)$. Social novelty index of WT mice is near 1 , indicating more time spent in S2 chamber with a novel mouse than in S1 chamber with the familiar mouse. The social novelty index of $\mathrm{KO}$ mice is near 0.5 , indicating a deficit in social memory.

Anxiety and hyperexcitability were assessed in Group $1 \mathrm{KO}$ mice and their controls using an open field test (Fig. $7 F$ ) by determining the time mice spent in thigmotaxis (near the walls) and the average velocity of the mice. Both control and $\mathrm{KO}$ mice exhibited similar time spent in thigmotaxis (Fig. 7G; Extended Data Fig. $7-4 ; t$ test, $\left.t_{(15)}=0.3455, p=0.7345\right)$, indicating no changes in anxious behavior following ephrin-B1 KO in astrocytes. Average velocity across the entire test was also similar between control and $\mathrm{KO}$ mice (Fig. 7H; Extended Data Fig. 7-4; $t$ test, $\left.t_{(15)}=0.1 .214, p=0.2435\right)$, suggesting no signs of hyperactivity.

\section{Discussion}

Interactions between neurons and astrocytes are essential for proper circuit formation, particularly during early postnatal development when synapses are rapidly forming and being eliminated. The studies presented here suggest that astrocytic ephrin-B1 regulates hippocampal $\mathrm{E} / \mathrm{I}$ balance by negatively influencing excitatory synapse formation and enhancing inhibition during early postnatal development. First, we found that loss of ephrin-B1 in astrocytes enhances excitability of CA1 hippocampal neurons. We observed increased evoked AMPAR- and NMDAR-mediated responses, increased excitatory synapse numbers, and higher dendritic spines density in CA1 neurons following the deletion of ephrin-B1 from astrocytes during P14-P28. In contrast, $\mathrm{OE}$ of astrocytic ephrin-B1 during the same period resulted in reduced evoked AMPAR- and NMDAR-mediated EPSCs. Second, evoked inhibitory responses were decreased in CA1 neurons of $\mathrm{KO}$ mice, most likely because of lower density of PV-expressing inhibitory neurons and a reduction of inhibitory synapses on CA1 pyramidal neurons in the SP and SO areas. In contrast, evoked IPSCs were enhanced on excitatory CA1 pyramidal cells following ephrin-B1 OE in developing astrocytes. Third, loss of astrocytic ephrin-B1 during postnatal development impaired mouse social behaviors. Together, these studies implicate astrocytic ephrin-B1 in developmental refinement of neuronal circuits.

Eph/ephrin signaling has been shown to modulate synapse development both in vitro and in vivo (Ethell et al., 2001; Henderson et al., 2001; Takasu et al., 2002; Henkemeyer et al., 2003; Liebl et al., 2003). Eph/ephrin-B signaling is involved in both presynaptic and postsynaptic differentiation and function. Presynaptic ephrin-Bs, specifically ephrin-B1 and -B2, interact with postsynaptic EphB2 to induce formation of functional presynaptic release sites on axons (Kayser et al., 2006; McClelland et al., 2009). Postsynaptically, Eph/ephrin-B signaling can affect synaptic plasticity, spinogenesis, glutamate receptor recruitment,
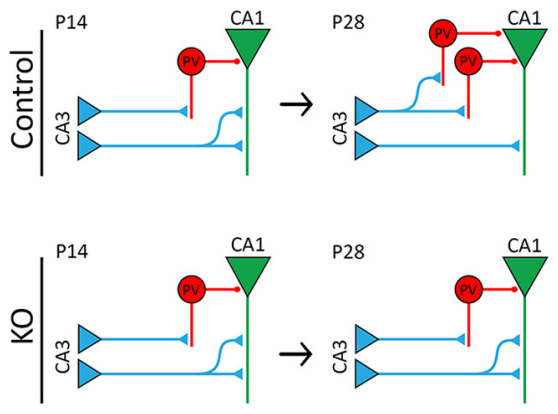

Figure 8. Model schematic of hippocampal circuitry in control and KO mice. Top, During P14-P28, excitatory synapse formation on CA1 hippocampal neurons is reduced, allowing for maturation and refinement of synaptic circuits through synapse elimination, while PV expression increases, allowing for proper inhibition of excitatory cells. Bottom, Deletion of astrocytic ephrin-B1 affects E/I balance in the developing hippocampus, resulting in a higher number of excitatory synapses onto CA1 pyramidal cells and reduced number of PV-expressing neurons in P28 hippocampus, thereby causing overall enhanced excitatory activity in the CA1 hippocampal pyramidal cells.

and synapse density (Dalva et al., 2000; Ethell et al., 2001; Grunwald et al., 2001, 2004; Contractor et al., 2002; Henkemeyer et al., 2003; McClelland et al., 2010; Xu et al., 2011). Eph/ephrin$\mathrm{B}$ signaling is also implicated in synaptic function (Essmann et al., 2008). However, the mechanism of astrocyte-neuron Eph/ ephrin-B signaling may differ from neuron-neuron signaling. In contrast to neuronal Eph/ephrin-B signaling, we observed enhanced excitatory synapse number and function with the loss of astrocytic ephrin-B1 and, conversely, reduced excitatory synapse numbers following the $\mathrm{OE}$ of ephrin-B1. It is possible that astrocytic ephrin-B1 can inhibit synapse formation by interfering with the interactions between axon terminals of CA3 neurons and postsynaptic dendrites of CA1 neurons. Indeed, we observed a redistribution of neuronal ephrin-B1 from cell body of CA1 neurons to dendrites following the deletion of ephrin-B1 from astrocytes, potentially increasing its interactions with EphB receptors at CA3 neuron terminals. Astrocytic ephrin-B1 can also induce removal of excess synapses via phagocytic mechanisms (Gong et al., 2019). As astrocytes are involved in synapse elimination via phagocytosis (Chung et al., 2013), astrocytic elimination of excess synapses may be mediated by Eph/ephrin signaling during the early postnatal development. Interestingly, despite increased excitatory synapse number, loss of ephrin-B1 in developing astrocytes does not affect AMPAR/NMDAR EPSC ratio and the levels of synaptic AMPARs, suggesting no changes in functional synapse maturation. However, loss of ephrin-B1 in astrocytes during development may result in delayed structural maturation as $\mathrm{KO}$ neurons have significantly more spines with smaller heads.

In addition, decreased inhibitory transmission in CA1 pyramidal neurons may also contribute to their enhanced excitability in astrocyte-specific ephrin-B1 KO mice. Astrocytes cocultured with developing neurons have been shown to significantly increase GABAergic synaptogenesis (Hughes et al., 2010) and increase amplitude and density of $\mathrm{GABA}_{\mathrm{A}}$ currents (Liu et al., 1996; Elmariah et al., 2005). Interestingly, neuronal cell bodies that were in direct contact with astrocytes exhibited higher amplitude and density of $\mathrm{GABA}_{\mathrm{A}}$ current (Liu et al., 1996). The increase in number of inhibitory presynaptic terminals, frequency of mIPSCs, and synaptic localization of $\mathrm{GABA}_{\mathrm{A}}$ receptor clusters was observed in neuronal cocultures with astrocytes (Elmariah et al., 2005) but was not mediated by astrocyte-derived thrombospondins (Hughes et al., 2010). Our studies show that 
the loss of astrocytic ephrin-B1 in the developing hippocampus leads to decreased inhibitory responses in CA1 hippocampal neurons potentially because of a decreased number of PVexpressing inhibitory neurons (Fig. 8). Additionally, we observed a reduced number of excitatory vGlut1-positive puncta on PV cells in CA1 hippocampus of $\mathrm{KO}$ mice, suggesting decreased excitatory drive onto PV-expressing neurons, which may lead to a reduced expression of $\mathrm{PV}$, therefore affecting their functions. Ephrin-B has been implicated in the neurogenesis of inhibitory neurons in the hippocampus (Talebian et al., 2018). Deletion of ephrin-B during embryogenesis affects the migration of interneurons, thereby reducing the number of interneurons and increasing the excitation of cortical networks (Talebian et al., 2017). Inhibitory neurons, in particular PV-expressing interneurons, are generated during embryonic development (Butt et al., 2005; Miyoshi et al., 2010; Tricoire et al., 2011) and migrate into the hippocampus by E14 (Tricoire et al., 2011). However, the expression of PV in interneurons is minimal until P12 and gradually increases until P30 (Nitsch et al., 1990; de Lecea et al., 1995), which coincides with the maturation of inhibitory neurons in rat hippocampus (Michelson and Lothman, 1989). In our study, ephrin-B1 was deleted from astrocytes during P14-P28 of $\mathrm{PV}$ cell maturation. As expression of PV is still increasing during this period in an activity-dependent manner, the loss astrocytic ephrin-B1 may be affecting the maturation of inhibitory circuits in the CA1 hippocampus by influencing excitatory innervation of PV inhibitory neurons (Fig. 8).

Although astrocytic ephrin-B1 may play a similar role as a negative regulator of excitatory synapse formation during development and adulthood (Koeppen et al., 2018), its role in inhibitory neurons is different in developing and adult hippocampus. In contrast to early postnatal development, deletion of astrocytic ephrin-B1 in the adult hippocampus does not affect IPSCs (Nguyen et al., 2020). In the adult hippocampus, inhibitory neurons are mature and have distinct properties (Wamsley and Fishell, 2017); therefore, fully established inhibitory neurons may require different astrocytic signaling for proper function. The exact role of Eph/ephrin signaling in interneurons is still unknown; further investigation is required to determine how Eph/ephrin signaling mediates interneuron maturation and function, and specifically how astrocytes may contribute to these mechanisms. Interestingly, ephrin-B signaling in astrocytes is shown to regulate neurogenesis in the dentate gyrus (DG) of the hippocampus (Ashton et al., 2012). EphB1 is also found to regulate cell number, proliferation, and positioning of neural stem and progenitor cells in the DG (Chumley et al., 2007). However, ephrin-B1 OE only in CA1 hippocampal astrocytes during P14P28 resulted in increased evoked IPSCs recorded from CA1 neurons, suggesting that the changes that we observe in our study are independent of neuronal differentiation in DG. Therefore, astrocytic ephrin-B1 may be essential in maintaining proper E/I balance by influencing PV cell development in the developing hippocampus. Furthermore, we observed the effects of astrocytic ephrin-B1 deletion on inhibitory function during P14-P28, but not in the adult hippocampus, suggesting the role of astrocytic ephrin-B1 in the maturation of inhibitory circuits in the developing hippocampus through yet unknown mechanisms.

We report that the loss of astrocytic ephrin-B1 enhanced excitatory function while also reducing inhibition. This E/I imbalance has been implicated in impaired sociability and social preference in the ASD mouse model (Lee et al., 2017). Aberrant synaptogenesis has been linked to several neurodevelopmental disorders, such as ASD and epilepsy (Huttenlocher and
Dabholkar, 1997; Lillis et al., 2015; Shen et al., 2016). Additionally, PV interneurons have been shown to have tight control over excitatory cell firing rhythms as PV interneurons can generate highly synchronized and fast inhibitory patterns ( $\mathrm{Hu}$ et al., 2014). Loss of PV interneurons may further contribute to the E/I imbalance and impaired social preference seen in astrocyte-specific ephrin-B1 KO mice. Indeed, loss of PV interneurons results in behavioral changes in mice similar core autism symptoms, including reduced social interactions and ultrasonic vocalizations, increased repetitive and stereotyped patterns of behaviors, impaired reversal learning, and increased seizure susceptibility (Wohr et al., 2015). It is interesting to note that these PVdepleted mice exhibited no impairments with motor function and no anxiety-like or depression-like behaviors (Wohr et al., 2015). Blocking synaptic transmission of PV neurons specifically in the ventral hippocampus was also shown to impair social memory discrimination (Deng et al., 2019). Our findings show that loss of astrocytic ephrin-B1 reduced sociability but had no effect on anxiety or motor function. The observed reduction in density of PV-expressing neurons in astrocyte-specific ephrin-B1 $\mathrm{KO}$ mice is most likely not specific to the hippocampus as we also see the changes in social behaviors. Together, this suggests that targeting astrocytic ephrin-B1 may be a potential avenue to repair PV cell functions and restore E/I balance in neurodevelopmental disorders.

The studies presented here suggest that astrocytic ephrin-B1 regulates $\mathrm{E} / \mathrm{I}$ balance in the CA1 hippocampus during early postnatal development. During the P14-P28 developmental period, astrocytic ephrin-B1 negatively regulates excitatory synapse formation, as deletion of ephrin-B1 in astrocytes results in increased excitation (Fig. 8), whereas OE of ephrin-B1 in astrocytes decreases excitation of CA1 neurons potentially through enhanced synapse elimination. Astrocytic ephrin-B1 may also affect inhibitory neuron maturation and function as loss of astrocytic ephrin-B1 reduces density of PV-expressing inhibitory neurons in the CA1 hippocampus, and in turn impairs evoked IPSCs and mIPSC amplitude recorded from CA1 pyramidal neurons. Conversely, OE of ephrin-B1 in astrocytes enhances evoked IPSCs in CA1 neurons. The deregulation of E/I balance in astrocyte-specific ephrin-B1 $\mathrm{KO}$ mice may contribute to observed changes in social behaviors of the mice. Genetic studies have linked mutations associated with Eph/ephrin signaling with neurodevelopmental disorders, including ASDs (Sanders et al., 2012; Robichaux et al., 2014), which are associated with social impairments and repetitive behaviors, as well as increased seizure susceptibility because of E/I imbalance (Gao and Penzes, 2015). Therefore, further understanding the role of astrocytic ephrin-B1 in establishing proper E/I balance during development may provide a potential target for treating neurodevelopmental disorders.

\section{References}

Allen NJ, Eroglu C (2017) Cell biology of astrocyte-synapse interactions. Neuron 96:697-708.

Allen NJ, Bennett ML, Foo LC, Wang GX, Chakraborty C, Smith SJ, Barres BA (2012) Astrocyte glypicans 4 and 6 promote formation of excitatory synapses via GluA1 AMPA receptors. Nature 486:410-414.

Araque A, Parpura V, Sanzgiri RP, Haydon PG (1999) Tripartite synapses: glia, the unacknowledged partner. Trends Neurosci 22:208-215.

Ashton RS, Conway A, Pangarkar C, Bergen J, Lim KI, Shah P, Bissell M, Schaffer DV (2012) Astrocytes regulate adult hippocampal neurogenesis through ephrin-B signaling. Nat Neurosci 15:1399-1406.

Beattie EC, Stellwagen D, Morishita W, Bresnahan JC, Ha BK, Von Zastrow M, Beattie MS, Malenka RC (2002) Control of synaptic strength by glial TNFalpha. Science 295:2282-2285. 
Bonansco C, Fuenzalida M (2016) Plasticity of hippocampal excitatory-inhibitory balance: missing the synaptic control in the epileptic brain. Neural Plast 2016:8607038.

Bush JO, Soriano P (2009) Ephrin-B1 regulates axon guidance by reverse signaling through a PDZ-dependent mechanism. Genes Dev 23:1586-1599.

Butt SJ, Fuccillo M, Nery S, Noctor S, Kriegstein A, Corbin JG, Fishell G (2005) The temporal and spatial origins of cortical interneurons predict their physiological subtype. Neuron 48:591-604.

Castaneda-Castellanos DR, Flint AC, Kriegstein AR (2006) Blind patch clamp recordings in embryonic and adult mammalian brain slices. Nat Protoc 1:532-542.

Christopherson KS, Ullian EM, Stokes CC, Mullowney CE, Hell JW, Agah A, Lawler J, Mosher DF, Bornstein P, Barres BA (2005) Thrombospondins are astrocyte-secreted proteins that promote CNS synaptogenesis. Cell 120:421-433.

Chumley MJ, Catchpole T, Silvany RE, Kernie SG, Henkemeyer M (2007) EphB receptors regulate stem/progenitor cell proliferation, migration, and polarity during hippocampal neurogenesis. J Neurosci 27:1348113490.

Chung WS, Clarke LE, Wang GX, Stafford BK, Sher A, Chakraborty C, Joung J, Foo LC, Thompson A, Chen C, Smith SJ, Barres BA (2013) Astrocytes mediate synapse elimination through MEGF10 and MERTK pathways. Nature 504:394-400.

Clarke LE, Barres BA (2013) Emerging roles of astrocytes in neural circuit development. Nat Rev Neurosci 14:311-321.

Contractor A, Rogers C, Maron C, Henkemeyer M, Swanson GT, Heinemann SF (2002) Trans-synaptic Eph receptor-ephrin signaling in hippocampal mossy fiber LTP. Science 296:1864-1869.

Dalva MB, Takasu MA, Lin MZ, Shamah SM, Hu L, Gale NW, Greenberg ME (2000) EphB receptors interact with NMDA receptors and regulate excitatory synapse formation. Cell 103:945-956.

de Lecea L, del Río JA, Soriano E (1995) Developmental expression of parvalbumin mRNA in the cerebral cortex and hippocampus of the rat. Brain Res Mol Brain Res 32:1-13.

Deng X, Gu L, Sui N, Guo J, Liang J (2019) Parvalbumin interneuron in the ventral hippocampus functions as a discriminator in social memory. Proc Natl Acad Sci USA 116:16583-16592.

Elmariah SB, Oh EJ, Hughes EG, Balice-Gordon RJ (2005) Astrocytes regulate inhibitory synapse formation via Trk-mediated modulation of postsynaptic GABAA receptors. J Neurosci 25:3638-3650.

Essmann CL, Martinez E, Geiger JC, Zimmer M, Traut MH, Stein V, Klein R, Acker-Palmer A (2008) Serine phosphorylation of ephrinB2 regulates trafficking of synaptic AMPA receptors. Nat Neurosci 11:1035-1043.

Ethell IM, Irie F, Kalo MS, Couchman JR, Pasquale EB, Yamaguchi Y (2001) EphB/syndecan-2 signaling in dendritic spine morphogenesis. Neuron 31:1001-1013.

Fellin T, Pascual O, Gobbo S, Pozzan T, Haydon PG, Carmignoto G (2004) Neuronal synchrony mediated by astrocytic glutamate through activation of extrasynaptic NMDA receptors. Neuron 43:729-743.

Fritschy JM (2008) Epilepsy, E/I balance and GABA(A) receptor plasticity. Front Mol Neurosci 1:5.

Gao R, Penzes P (2015) Common mechanisms of excitatory and inhibitory imbalance in schizophrenia and autism spectrum disorders. Curr Mol Med 15:146-167.

Gong J, Gaitanos TN, Luu O, Huang Y, Gaitanos L, Lindner J, Winklbauer R, Klein R (2019) Gulp1 controls Eph/ephrin trogocytosis and is important for cell rearrangements during development. J Cell Biol 218:3455-3471.

Grunwald IC, Korte M, Wolfer D, Wilkinson GA, Unsicker K, Lipp HP, Bonhoeffer T, Klein R (2001) Kinase-independent requirement of EphB2 receptors in hippocampal synaptic plasticity. Neuron 32:1027-1040.

Grunwald IC, Korte M, Adelmann G, Plueck A, Kullander K, Adams RH, Frotscher M, Bonhoeffer T, Klein R (2004) Hippocampal plasticity requires postsynaptic ephrinBs. Nat Neurosci 7:33-40.

Hama H, Hara C, Yamaguchi K, Miyawaki A (2004) PKC signaling mediates global enhancement of excitatory synaptogenesis in neurons triggered by local contact with astrocytes. Neuron 41:405-415.

Henderson JT, Georgiou J, Jia Z, Robertson J, Elowe S, Roder JC, Pawson T (2001) The receptor tyrosine kinase EphB2 regulates NMDA-dependent synaptic function. Neuron 32:1041-1056.

Henkemeyer M, Itkis OS, Ngo M, Hickmott PW, Ethell IM (2003) Multiple EphB receptor tyrosine kinases shape dendritic spines in the hippocampus. J Cell Biol 163:1313-1326.
Henneberger C, Papouin T, Oliet SH, Rusakov DA (2010) Long-term potentiation depends on release of D-serine from astrocytes. Nature 463:232236.

Hitti FL, Siegelbaum SA (2014) The hippocampal CA2 region is essential for social memory. Nature 508:88-92.

Hollingsworth EB, McNeal ET, Burton JL, Williams RJ, Daly JW, Creveling CR (1985) Biochemical characterization of a filtered synaptoneurosome preparation from guinea pig cerebral cortex: cyclic adenosine $3^{\prime}: 5^{\prime}$ monophosphate-generating systems, receptors, and enzymes. J Neurosci 5:2240-2253.

$\mathrm{Hu}$ H, Gan J, Jonas P (2014) Interneurons. Fast-spiking, parvalbumin(+) GABAergic interneurons: from cellular design to microcircuit function. Science 345:1255263.

Hughes EG, Elmariah SB, Balice-Gordon RJ (2010) Astrocyte secreted proteins selectively increase hippocampal GABAergic axon length, branching, and synaptogenesis. Mol Cell Neurosci 43:136-145.

Hussain NK, Thomas GM, Luo J, Huganir RL (2015) Regulation of AMPA receptor subunit GluA1 surface expression by PAK3 phosphorylation. Proc Natl Acad Sci USA 112:E5883-E5890.

Huttenlocher PR, Dabholkar AS (1997) Regional differences in synaptogenesis in human cerebral cortex. J Comp Neurol 387:167-178.

Kaidanovich-Beilin O, Lipina T, Vukobradovic I, Roder J, Woodgett JR (2011) Assessment of social interaction behaviors. J Vis Exp 48:2473.

Kayser MS, McClelland AC, Hughes EG, Dalva MB (2006) Intracellular and trans-synaptic regulation of glutamatergic synaptogenesis by EphB receptors. J Neurosci 26:12152-12164.

Ko J (2017) Neuroanatomical substrates of rodent social behavior: the medial prefrontal cortex and its projection patterns. Front Neural Circuits 11:41.

Koeppen J, Nguyen AQ, Nikolakopoulou AM, Garcia M, Hanna S, Woodruff S, Figueroa Z, Obenaus A, Ethell IM (2018) Functional consequences of synapse remodeling following astrocyte-specific regulation of Ephrin-B1 in the adult hippocampus. J Neurosci 38:5710-5726.

Kucukdereli H, Allen NJ, Lee AT, Feng A, Ozlu MI, Conatser LM, Chakraborty C, Workman G, Weaver M, Sage EH, Barres BA, Eroglu C (2011) Control of excitatory CNS synaptogenesis by astrocyte-secreted proteins Hevin and SPARC. Proc Natl Acad Sci USA 108:E440-E449.

Lee E, Lee J, Kim E (2017) Excitation/inhibition imbalance in animal models of autism spectrum disorders. Biol Psychiatry 81:838-847.

Liebl DJ, Morris CJ, Henkemeyer M, Parada LF (2003) mRNA expression of ephrins and Eph receptor tyrosine kinases in the neonatal and adult mouse central nervous system. J Neurosci Res 71:7-22.

Lillis KP, Wang Z, Mail M, Zhao GQ, Berdichevsky Y, Bacskai B, Staley KJ (2015) Evolution of network synchronization during early epileptogenesis parallels synaptic circuit alterations. J Neurosci 35:9920-9934.

Liu QY, Schaffner AE, Li YX, Dunlap V, Barker JL (1996) Upregulation of GABAA current by astrocytes in cultured embryonic rat hippocampal neurons. J Neurosci 16:2912-2923.

Lovelace JW, Rais M, Palacios AR, Shuai XS, Bishay S, Popa O, Pirbhoy PS, Binder DK, Nelson DL, Ethell IM, Razak KA (2020) Deletion of Fmr1 from forebrain excitatory neurons triggers abnormal cellular, EEG, and behavioral phenotypes in the auditory cortex of a mouse model of Fragile X syndrome. Cereb Cortex 30:969-988.

Mayford M, Siegelbaum SA, Kandel ER (2012) Synapses and memory storage. Cold Spring Harb Perspect Biol 4:a005751.

McClelland AC, Sheffler-Collins SI, Kayser MS, Dalva MB (2009) Ephrin-B1 and ephrin-B2 mediate EphB-dependent presynaptic development via syntenin-1. Proc Natl Acad Sci USA 106:20487-20492.

McClelland AC, Hruska M, Coenen AJ, Henkemeyer M, Dalva MB (2010) Trans-synaptic EphB2-ephrin-B3 interaction regulates excitatory synapse density by inhibition of postsynaptic MAPK signaling. Proc Natl Acad Sci USA 107:8830-8835.

Michelson HB, Lothman EW (1989) An in vivo electrophysiological study of the ontogeny of excitatory and inhibitory processes in the rat hippocampus. Brain Res Dev Brain Res 47:113-122.

Miyoshi G, Hjerling-Leffler J, Karayannis T, Sousa VH, Butt SJ, Battiste J, Johnson JE, Machold RP, Fishell G (2010) Genetic fate mapping reveals that the caudal ganglionic eminence produces a large and diverse population of superficial cortical interneurons. J Neurosci 30:1582-1594.

Moeller ML, Shi Y, Reichardt LF, Ethell IM (2006) EphB receptors regulate dendritic spine morphogenesis through the recruitment/phosphorylation of focal adhesion kinase and RhoA activation. J Biol Chem 281:15871598 . 
Nguyen AQ, Koeppen J, Woodruff S, Mina K, Figueroa Z, Ethell IM (2020) Astrocytic ephrin-B1 controls synapse formation in the hippocampus during learning and memory. Front Synaptic Neurosci 12:10.

Nitsch R, Soriano E, Frotscher M (1990) The parvalbumin-containing nonpyramidal neurons in the rat hippocampus. Anat Embryol 181:413-425.

Nolt MJ, Lin Y, Hruska M, Murphy J, Sheffler-Colins SI, Kayser MS, Passer J, Bennett MV, Zukin RS, Dalva MB (2011) EphB controls NMDA receptor function and synaptic targeting in a subunit-specific manner. J Neurosci 31:5353-5364.

Nygaard KR, Maloney SE, Dougherty JD (2019) Erroneous inference based on a lack of preference within one group: autism, mice, and the social approach task. Autism Res 12:1171-1183.

Robichaux MA, Chenaux G, Ho HY, Soskis MJ, Dravis C, Kwan KY, Sestan N, Greenberg ME, Henkemeyer M, Cowan CW (2014) EphB receptor forward signaling regulates area-specific reciprocal thalamic and cortical axon pathfinding. Proc Natl Acad Sci USA 111:2188-2193.

Sanders SJ, Murtha MT, Gupta AR, Murdoch JD, Raubeson MJ, Willsey AJ, Ercan-Sencicek AG, DiLullo NM, Parikshak NN, Stein JL, Walker MF, Ober GT, Teran NA, Song Y, El-Fishawy P, Murtha RC, Choi M, Overton JD, Bjornson RD, Carriero NJ, et al. (2012) De novo mutations revealed by whole-exome sequencing are strongly associated with autism. Nature 485:237-241.

Segura I, Essmann CL, Weinges S, Acker-Palmer A (2007) Grb4 and GIT1 transduce ephrinB reverse signals modulating spine morphogenesis and synapse formation. Nat Neurosci 10:301-310.

Shen Y, Qin H, Chen J, Mou L, He Y, Yan Y, Zhou H, Lv Y, Chen Z, Wang J, Zhou YD (2016) Postnatal activation of TLR4 in astrocytes promotes excitatory synaptogenesis in hippocampal neurons. J Cell Biol 215:719734.

Sloniowski S, Ethell IM (2012) Looking forward to EphB signaling in synapses. Semin Cell Dev Biol 23:75-82.

Stellwagen D, Malenka RC (2006) Synaptic scaling mediated by glial TNFalpha. Nature 440:1054-1059.

Südhof TC, Malenka RC (2008) Understanding synapses: past, present, and future. Neuron 60:469-476.
Takasu MA, Dalva MB, Zigmond RE, Greenberg ME (2002) Modulation of NMDA receptor-dependent calcium influx and gene expression through EphB receptors. Science 295:491-495.

Talebian A, Britton R, Ammanuel S, Bepari A, Sprouse F, Birnbaum SG, Szabo G, Tamamaki N, Gibson J, Henkemeyer M (2017) Autonomous and non-autonomous roles for ephrin-B in interneuron migration. Dev Biol 431:179-193.

Talebian A, Britton R, Henkemeyer M (2018) Abnormalities in cortical interneuron subtypes in ephrin-B mutant mice. Eur J Neurosci 48:1803-1817.

Tricoire L, Pelkey KA, Erkkila BE, Jeffries BW, Yuan X, McBain CJ (2011) A blueprint for the spatiotemporal origins of mouse hippocampal interneuron diversity. J Neurosci 31:10948-10970.

Ullian EM, Sapperstein SK, Christopherson KS, Barres BA (2001) Control of synapse number by glia. Science 291:657-661.

Wamsley B, Fishell G (2017) Genetic and activity-dependent mechanisms underlying interneuron diversity. Nat Rev Neurosci 18:299-309.

Wohr M, Orduz D, Gregory P, Moreno H, Khan U, Vorckel KJ, Wolfer DP, Welzl H, Gall D, Schiffmann SN, Schwaller B (2015) Lack of parvalbumin in mice leads to behavioral deficits relevant to all human autism core symptoms and related neural morphofunctional abnormalities. Transl Psychiatry 5:e525.

Xu NJ, Henkemeyer M (2012) Ephrin reverse signaling in axon guidance and synaptogenesis. Semin Cell Dev Biol 23:58-64.

Xu NJ, Sun S, Gibson JR, Henkemeyer M (2011) A dual shaping mechanism for postsynaptic ephrin-B3 as a receptor that sculpts dendrites and synapses. Nat Neurosci 14:1421-1429.

Yan QJ, Rammal M, Tranfaglia M, Bauchwitz RP (2005) Suppression of two major Fragile X syndrome mouse model phenotypes by the mGluR5 antagonist MPEP. Neuropharmacology 49:1053-1066.

Yan QJ, Asafo-Adjei PK, Arnold HM, Brown RE, Bauchwitz RP (2004) A phenotypic and molecular characterization of the fmrl-tm1Cgr fragile X mouse. Genes Brain Behav 3:337-359.

Zimmer M, Palmer A, Kohler J, Klein R (2003) EphB-ephrinB bi-directional endocytosis terminates adhesion allowing contact mediated repulsion. Nat Cell Biol 5:869-878. 